\title{
In der Grauzone: Akkreditierung zwischen öffentlich-rechtlicher und privatrechtlicher Rechtsdurchsetzung
}

\author{
Hans-Heinrich Trute"
}

\section{A. Einleitung}

Die private Durchsetzung von öffentlich-rechtlichen Normen und Standards erfasst eine Vielzahl von sehr heterogenen und durchaus nicht notwendig neuen Konstellationen. ${ }^{1}$ Schon der im Gefolge der europäischen Dienstleistungsrichtlinie zunehmende Verzicht auf präventive Verwaltungskontrollen überantwortet die Durchsetzung des materiellen Rechts zumindest partiell den Privaten. Ohne eine Änderung der materiell-rechtlichen Standards wird auf diese Weise eine andere Vollzugsrealität geschaffen. ${ }^{2}$ Die Konstellationen reichen von der Einschaltung Privater in staatliche Verfahrenszusammenhänge, ${ }^{3}$ insbesondere zur Wissensgenerierung, der Aktualisierung bestimmter Organisationspflichten und Schaffung von Kontrollorganen in privaten Einrichtungen, ${ }^{4}$ über die Institutionalisierung von Privaten als Kontrolleure oder die Begründung von Einflusspositionen zur Wahrung öffentlicher Interessen ${ }^{5}$ bis hin zu Formen der Verstärkung und Abstimmung von öffentlich- rechtlicher und privater Rechtsdurchsetzung, wie etwa im Kartellrecht. ${ }^{6}$ Die Gründe dafür sind vielfältig: Staatsentlastung, Schaffung von mehr Staatsferne bei der Wahrnehmung bestimmter Aufgaben, Wissensgenerierung durch Expertise, Schaffung von vertrau-

* Prof. Dr. Hans-Heinrich Trute lehrt Öffentliches Recht, Medien- und Telekommunikationsrecht an der Universität Hamburg. Der Verfasser hat in dem Vorlageverfahren des VG Arnsberg beim Bundesverfassungsgericht in Sachen Akkreditierung eine Stellungnahme für eine Akkreditierungsagentur abgeben und ist auch gutachtlich in einem Teilbereich tätig gewesen.

1 Dazu aus der Fülle der Literatur die Beiträge in W. Hoffmann-Riem/E. Schmidt-Aßmann (Hrsg.), Öffentliches Recht und Privatrecht als wechselseitige Auffangordnung, Baden-Baden 1996; M. Burgi, Funktionale Privatisierung und Verwaltungshilfe, Tübingen 1999; M. Knauff, Der Gewährleistungsstaat: Reform der Daseinsvorsorge, Berlin 2004; Cl. Franzius, Gewährleistung im Recht, Tübingen 2009; H. Schulze-Fielitz, Grundmodi der Aufgabenwahrnehmung, in: W Hoffmann-Riem/E. Schmidt-Aßmann/A. Voßkuhle (Hrsg.), Grundlagen des Verwaltungsrechts, Bd. $1^{2}$ München 2012, Rn. $91 \mathrm{ff}$.

2 Dazu P. Cancik, Fingierte Rechtsdurchsetzung, DÖV 2011, S. $1 \mathrm{ff}$.

3 Dazu A.Seidel, Privater Sachverstand und staatliche Garantenstellung im Verwaltungsverfahren, München 2000; ausführlich zum geregelten Umgang mit verwaltungsexterner Expertise I. Augsberg, Informationsverwaltungsrecht, Tübingen 2014, S. $115 \mathrm{ff}$.

4 Dazu demnächst ausführlich A.-K. Lydia Reiling, Der Hybride - Administrative Wissensorganisation im privaten Bereich, Diss. Konstanz 2014.

5 Vgl. etwa die Versuche, Internetprovider zur Blockierung von rechtswidrigen Angeboten in die Lage zu versetzen, etwa infolge des Art. 23 Abs. 5 lit a. des „Proposal for a regulation of the European Parliament and the Council laying down measures concerning the European single market for electronic communications and to achieve a Connected Continent, amending Directives 2002/20/EC, 2002/21/EC and 2002/22/EC and Regulations (EC) No 1211/2009 and (EU) No 531/2012, COM (2013) 627 final " und die Bedenken etwa in der Stellungnahme des Bundesrates, Empfehlung des Bundesrates v. 18.11.13, Drucksache 689/1/13, S. 11; zu anderen Aspekten der Verantwortlichkeit von Intermediären im Internet vgl. A. Lauber-Rönsberg, Rechtsdurchsetzung bei Persönlichkeitsrechtsverletzungen im Internet - Verantwortlichkeit von Intermediären und Nutzern in Meinungsforen und Personenbewertungsportalen, MMR 2014, S. $210 \mathrm{ff}$.

6 R. Koch, Rechtsdurchsetzung im Kartellrecht: Public vs. private enforcement. JZ 2013, S. 390 ff. 
ensgenerierenden Institutionen in transnationalen Zusammenhängen, Europäisierung und Internationalisierung, um nur einige der Stichworte zu nennen. Wie immer einzelne der Konstellationen rechtspolitisch oder verwaltungswissenschaftlich zu bewerten sein mögen, der Sache nach geht es selten noch um die Alternative der öffentlich-rechtlichen oder privatrechtlichen Verfassung des jeweiligen institutionellen Arrangements, sondern um die angemessene rechtliche Durchformung einer hybriden, staatliche und private Akteure umfassenden Konstellation, die sicherstellt, dass die Aufgabenwahrnehmung im öffentlichen Interesse erfolgt. ${ }^{7}$ Insoweit kommt es vor allem darauf an, innerhalb einer Regelungsstruktur die gemeinwohlbezogenen Anforderungen zur Geltung zu bringen.

Akkreditierung und Zertifizierung von Produkten, Systemen, Dienstleistungen, Einrichtungen und Personen ist eine der Formen, die in diesem Kontext eine prominente Rolle spielen. Sie finden sich mittlerweile in einer Vielzahl von Rechtsgebieten, zu nennen sind beispielsweise das Produktsicherheitsrecht ${ }^{8}$ das technische Sicher-

7 H. Schulze-Fielitz, Aufgabenwahrnehmung (Fn. 1), Rn. 118 ff.; E. Schmidt-Aßmann, Das allgemeine Verwaltungsrecht als Ordnungsidee, Berlin/Heidelberg/New York, 2004, 5. Kap. Rn. 53 ff., 6. Kap. Rn. 28 ff.; H.-H. Trute, Die Legitimation der öffentlichen Verwaltung, in: W Hoffmann-Riem/E. Schmidt-Aßmann/A. Voßkuhle (Hrsg.), Grundlagen (Fn. 1), $\int 6$ Rn. 89 ff.; ders., Gemeinwohlsicherungen im Gewährleistungsstaat, in, G. F. Schuppert/F. Neidhardt. Gemeinwohl. Auf der Suche nach Substanz, Berlin 2002, S. $329 \mathrm{ff}$.

8 Im Produktsicherheitsrecht kann die Akkreditierung und Zertifizierung auf eine vergleichsweise lange Entwicklung zurücksehen; H.C. Röhl, Akkreditierung und Zertifizierung im Produktsicherheitsrecht, Berlin/Heidelberg/New York 2000; ders., Konformitätsbewertung im Europäischen Produktsicherheitsrecht, in: E. Schmidt-Aßmann/ B. Schöndorf-Haubold, (Hrsg.), Der Europäische Verwaltungsverbund, Tübingen 2005, S. 153 ff.; K. Bieback, Zertifizierung und Akkreditierung. Das Zusammenwirken staatlicher und nichtstaatlicher Akteure in gestuften Prüfsystemen, Baden-Baden, 2008, S. 38 ff.; A.Kapoor/ Th. Klindt, Die Reform des Akkreditierungswesen im Europäischen Produktsicherheitsrecht, EuZW 20 (2009), S. 134 ff.; W.Tiede/ Ch. Ryczewski/ M. Yang, Einführung in das Akkreditierungsrecht Deutschlands, NVwZ 2012, 1212 ff.; G. Dimitropoulos, Zertifizierung und Akkreditierung im Internationalen Verwaltungsverbund, Tübingen 2012; H. Ischil Th. Zimmermann, Die Akkreditierung - eine volkswirtschaftliche Basisinfrastruktur, Die Volkswirtschaft 2011, 53 ff.; Durch die am 1.1.2010 in Kraft getretene VO EG Nr. 765/2008 des Europäischen Parlaments und des Rates über die Vorschriften für die Akkreditierung und Marktüberwachung von Produkten (ABlEU Nr. L 218/30 v. 13.8.2008) ist die Akkreditierung noch einmal harmonisiert worden. 


\section{heitsrecht, das Umweltrecht, ${ }^{9}$ das Ausbildungs- und Hochschulrecht ${ }^{10}$ und das So- zialrecht. ${ }^{11}$}

\section{Im Hochschulbereich kann die Akkreditierung von Akkreditierungsagenturen, Stu- diengängen und Qualitätssicherungssystemen mittlerweile jedenfalls auf eine Viel- zahl von durchgeführten Verfahren verweisen. ${ }^{12}$ Für den Wissenschaftsrat hat sie}

9 Verordnung (EG) Nr. 1221/2009 des Europäischen Parlamentes und des Rates vom 25. November 2009 über die freiwillige Teilnahme von Organisationen an einem Gemeinschaftssystem für Umweltmanagement und Umweltbetriebsprüfung und zur Aufhebung der Verordnung (EG) Nr. 761/2001, sowie der Beschlüsse der Kommission 2001/861/EG und 2006/193/EG, ABl. 2009 L 342/1. Umgesetzt durch das Gesetz zur Ausführung der Verordnung (EG) N. 1221/2009 des Europäischen Parlaments und des Rates v. 25. November 2009 über die freiwillige Teilnahme von Organisationen an einem Gemeinschaftssystems für Umweltmanagement und Umweltbetriebsprüfung zur Aufhebung der Verordnung (EG) Nr. 761/2001 sowie der Beschlüsse der Kommission 2001/681/EG und 2006/193/EG (Umweltauditgesetz -UAG). Umgesetzt durch das Gesetz zur Ausführung der Verordnung (EG) N. 1221/2009 des Europäischen Parlaments und des Rates v. 25. November 2009 über die freiwillige Teilnahme von Organisationen an einem Gemeinschaftssystems für Umweltmanagement und Umweltbetriebsprüfung zur Aufhebung der Verordnung (EG) Nr. 761/2001 sowie der Beschlüsse der Kommission 2001/681/EG und 2006/193/EG (Umweltauditgesetz -UAG); vgl. dazu ausführlich C. Leifer, Das europäische Umweltmanagementsystem EMAS als Element gesellschaftlicher Selbstregulierung, Tübingen 2007; Bieback, Zertifizierung (Fn. 8), S. 97 ff.; M. Langerfeldt, Das novellierte Environmental Management and Audit Scheme (EMAS-II) und sein Potential zur Privatisierung der umweltrechtlichen Betreiberüberwachung in Deutschland, Berlin 2007.

10 Aus der Vielzahl der Literatur: J. Lege, Die Akkreditierung von Studiengängen, JZ 2005, 698 ff.; H. Pünder, Zertifizierung und Akkreditierung - private Qualitätskontrolle unter staatlicher Gewährleistungsverantwortung, ZHR 170 (2006), S. 567 ff.; Ch. Heitsch, Verfassungs- und verwaltungsrechtliche Fragen der Akkreditierung von Studiengängen, DÖV 2007, 770 ff.; ders., Rechtsnatur der Akkreditierungsentscheidungen, WissR 42 (2009), S. 163 ff.; Th. Mann/ D. Immer, Rechtsprobleme der Akkreditierung von Studiengängen, RdJB 2007, 334 ff.; K. Bieback, Zertifizierung (Fn. 8), S. 132 ff.; M. Martini, Akkreditierung im Hochschulrecht - Institutionelle Akkreditierung, Programmakkreditierung, Prozessakkreditierung, WisssR 41 (2008), 232 ff.; K. Serrano-Vallarde, Evaluation, Akkreditierung und Politik, Wiesbaden 2008; R. Brinktrine, Akkreditierungsverfahren und -modelle nach Maßgabe des Hochschulrechts der Länder, WissR 42 (2009), 164 ff.; R. Müller-Terpitz, Verfassungsrechtliche Implikationen der Akkreditierungsverfahren, WissR 42 (2009) 116 ff.; U. Mager, Ist die Akkreditierung von Studiengängen an Hochschulen des Landes Baden-Württemberg verfassungsgemäß, VBlBW 2009, 9 ff.; K. Wilhelm, Verfassungs- und verwaltungsrechtliche Fragen der Akkreditierung von Studiengängen, 2009; Kl. Grünewald, Akkreditierung von Studiengängen nach nordrheinwestfälischem Hochschulrecht, NWVBl 2011, 132 ff.; L. Knopp, Akkreditierung im verfassungsrechtlichen Zwielicht, in: W.Ch. Zimmerli/ L. Knopp (Hrsg.), Freiheit von Kunst Wissenschaft, Forschung und Lehre - was heißt das nach Bologna, Baden-Baden 2012, S. 197ff.; A. Merschmann, Die Rechtsnatur der Akkreditierung von Studiengängen, NVwZ, 2011, 847 ff.; M. Siever, Qualitätssicherung durch Programm- und Systemakkreditierung im deutschen Hochschulsystem: unter besonderer Berücksichtigung der Rechtslage in Baden-Württemberg, Hamburg 2011; V. J. Voth, Akkreditierung im Hochschulsektor vor den Anforderungen der Wissenschaftsfreiheit, Diss. Hamburg, 2011; D. Immer, Rechtsprobleme der Akkreditierung von Studiengängen, Göttingen 2013.

11 Vgl. etwa ausführlich M. Banafsche, in: Gagel (Hrsg.), SGB III $\mathbb{1} 176$ Rn. 4 ff.

12 Die Zahl der von den Agenturen durchgeführten Verfahren belaufen sich Stand 2010 auf ca. 10.000 Verfahren; Wissenschaftsrat, Empfehlungen zur Akkreditierung als Instrument der Qualitätssicherung, Drs. 2259/12, S. 36. Es bestehen derzeit rund 7900 akkreditierte Studiengänge (Stand 5.5.2014), vgl. http://www.hs-kompass2.de/kompass/xml/akkr/akkr_stat. 
sich ungeachtet aller Kritik im Grundsatz als Instrument der Qualitätssicherung bewährt. ${ }^{13}$

Gleichwohl ist ihre Struktur in der verfassungsrechtlichen und verwaltungsrechtlichen Einordnung nach wie vor umstritten. Sie oszilliert zwischen der öffentlichrechtlichen und der privatrechtlichen Konstruktion der Akkreditierung von Studiengängen oder Qualitätssicherungssystemen und die Kritik changiert zum Teil zwischen Hochschulpolitik und rechtlicher Konstruktion. Zugleich wird die Diskussion nicht selten unbeeinflusst von der Diskussion um andere Kooperationsformen geführt. Betrachtet man die Kritik durch die Linse anderer Felder, in denen Modelle der Qualitätssicherung weiter entfaltet worden sind und damit auch bereits stabilere Formen gewonnen haben, dann erscheinen die Auseinandersetzungen eher auch Unsicherheiten über veränderte Formen staatlicher Aufgabenwahrnehmung im Kooperationsspektrum mit Privaten widerzuspiegeln.

Insoweit erweist sich das Thema der Akkreditierung als ein solches aus dem breiten Spektrum eines Formenwandels, dem, wie dargelegt, ganz unterschiedliche Phänomene zuzurechnen sind.

\section{B. Strukturelemente der Akkreditierung und Zertifizierung}

Das Produktsicherheitsrecht bildet das am weitesten ausgeformte System einer nationalen, europäischen und heute auch internationalen Akkreditierung und Zertifizierung, das freilich in seiner Entwicklung, zumal in Deutschland, auf ähnliche Konstruktionsschwierigkeiten zurückblicken kann, wie sie den Hochschulbereich derzeit kennzeichnen. ${ }^{14}$ Heute ist es allerdings ein europäisch und international weitgehend harmonisiertes Verfahren der Sicherung bestimmter Qualitätsstandards, ${ }^{15}$ dass durch ein Zusammenspiel von öffentlich-rechtlichen und privaten Elementen gekennzeichnet ist.

13 Vgl. dazu Wissenschaftsrat, Empfehlungen (Fn. 12), S. 49 ff. Die Kritik ist von gewissen strategischen Elementen nicht frei, die gelegentlich auf den Bologna-Prozess insgesamt zielt. Politik und Hochschule nutzen gerne wechselseitige Verantwortungszuweisungen und invisibilisieren dadurch ihren jeweiligen eigenen Beitrag zur Bürokratisierung des Modells. Denn es waren schließlich die von den Hochschulen, wenn auch auf der Basis der ländergemeinsamen Strukturvorgaben, selbst entwickelten Programme, die durch ein hohes Maß an bürokratischer Dichte und mangelnder Analyse der Studierbarkeit akkreditiert worden sind. Die bürokratische Parallelbürokratie, die von Seiten der Hochschulrektorenkonferenz heute beklagt wird, ist auch ein Ergebnis der hochschulinternen Wahrnehmung der Aufgaben, der von Seiten der Hochschuladministrationen nicht selten als bürokratischer Prozess angelegt worden war; zu Recht auf die unterschiedlichen Beiträge verweisend Wissenschaftsrat, Empfehlungen zur Akkreditierung als Instrument der Qualitätssicherung, Drs. 2259/12, S. 52. Es war im Übrigen die Hochschulrektorenkonferenz, die 1998 die Ablösung der staatlichen Genehmigungen auf der Grundlage von Rahmenprüfungsordnungen durch ein System der Akkreditierung forderte; vgl. HRK, Akkreditierungsverfahren. Entschließung des 185. Plenums v. 6. Juli 1998.

14 Auch hier war seinerzeit versucht worden, die benannten Stellen als Beliehene auszuweisen und damit ein staatlich dominiertes System zu schaffen bzw. beizubehalten, dass sich freilich unter dem Druck der europäischen Harmonisierung nicht mehr halten ließ; vgl. dazu H.C. Röhl, Konformitätsbewertung (Fn. 8), S. $153 \mathrm{ff}$.

15 Vgl. G. Dimitropoulos, Zertifizierung (Fn. 8), S. 38 ff. 
Ein solchermaßen europäisch und international harmonisiertes System lässt sich als staatliches System kaum sinnvoll konzipieren, sondern ist für seine Akzeptanz darauf angewiesen, dass vertrauenswürdige Institutionen international akzeptierte Standards applizieren. Insoweit kann die Bedeutung der Akkreditierung und Zertifizierung für eine funktionsfähige Volkswirtschaft angesichts der Fragmentierung der Wertschöpfungsketten gar nicht überschätzt werden. Die Akkreditierung und Zertifizierung signalisiert einen bestimmten Qualitätsstandard, auf den sich Abnehmer von Vorleistungen verlassen können sollen. Von daher wird in vielen Bereichen verlangt, dass Erbringer von Vorleistungen, seien es Produkte, Dienstleistungen, Tätigkeiten von Prüflaboratorien oder anderen Einrichtungen ein zertifiziertes Qualitätssicherheitssystem implementiert haben. ${ }^{16}$ Auf diese Weise soll ein Vertrauenssystem geschaffen werden, das eigene Überprüfungen erübrigen und die Anknüpfung an von aussen nur schwer beurteilbare Reputation ersetzen kann. Es ist schwer vorstellbar, wie ein internationalisiertes Netzwerk von Transaktionspartnern ohne solche vertrauensgenerierenden Institutionen sollte noch funktionieren können. ${ }^{17}$ Jedenfalls aber wären die Kosten der Transaktionen deutlich höher.

\section{Das Produktsicherheitsrecht}

Dieses System ist in seiner Funktionsweise eng mit einer international verflochtenen Wirtschaft verbunden, aber in seinen Grundelementen darauf nicht beschränkt, sondern gewinnt überall dort Bedeutung, wo Produktions- und Leistungsvorgänge nationale Grenzen überschreiten oder Leistungen angeboten werden, deren Qualität von den Abnehmern nur begrenzt eingeschätzt werden können. In diesen Fällen gewinnen Qualitätsnachweise eine Rolle, die nicht notwendig staatlich erbracht werden müssen, in denen der Staat aber eine Vertrauen generierende Prüfinfrastruktur einrichtet. Das System ist durch bestimmte materielle, institutionelle und prozedurale Elemente gekennzeichnet. Der Sache nach geht es stets darum, dass bestimmte öffentlich-rechtliche Normen und Standards nicht von der staatlichen Administration, sondern durch eine aufgefächerte Regelungsstruktur von staatlichen, hybriden und privaten Akteuren umgesetzt bzw. deren Beachtung bescheinigt wird.

Der Sache nach meint die Akkreditierung nach dem europäisch harmonisierten Rahmen ${ }^{18}$ die Bestätigung durch eine nationale Akkreditierungsstelle, dass eine Konfor-

16 Für den Lebensmittelbereich vgl. etwa Th. Claußen, in: R. Streinz (Hrsg.) Lebensmittelrechts-Handbuch, Stand 2013, Einleitung, Rn. 300 (354); D.Langner/ T.Klindt, in: M. A. Dauses(Hrsg.), EUWirtschaftsrecht, 2013, C.VI. Rdn. 29.

17 H. Ischil Th. Zimmermann, Volkswirtschaftliche Basisinfrastruktur (Fn. 8), S. 53 ff.

18 Der New Legislative Framework wird gebildet durch die VO (EG) 764/2008, die VO (EG) 765/2008 und den Beschluss 768/2008/EG. Zum New Legislative Framework als gemeinschaftlichem Rahmen der Vermarktung von Produkten vgl. G. Dimitropoulos, Zertifizierung (Fn. 8), S. 91 ff.; M. Banafsche (Fn. 11), SGB III $\mathbb{1} 176$ Rn. 4 ff.; D. Langer/Th. Klindt, Technische Sicherheitsvorschriften und Normen, in: Dauses (Hrsg.), Handbuch des EU-Wirtschaftsrechts, 35. EL 2014, C. VI.; U. Soltau/ C. Sternly, Neue Möglichkeiten für die Zertifizierung, Onkologie 35 (2012), S. 7 ff. 
mitätsbewertungsstelle die in harmonisierten Normen festgelegten Anforderungen und ggf. zusätzliche Anforderungen erfüllt, um eine spezielle Konformitätsbewertungstätigkeit durchzuführen. ${ }^{19}$ Konformitätsbewertung ist in diesem Sinne ein Verfahren zur Bewertung, ob spezifische Anforderungen an ein Produkt, ein Verfahren, eine Dienstleistung, ein System, eine Person oder eine Stelle erfüllt sind. ${ }^{20}$ Diese Bewertung der Übereinstimmung mit den Normen und Standards stellt ein zentrales und auch entsprechend in seiner Konzeption lange umstrittenes Element dar.

Eine typischerweise mit hoheitlichen Aufgaben betraute, oftmals beliehene Stelle lässt anhand europäischer ${ }^{21}$ oder nationaler Vorgaben eine oder mehrere (private) Einrichtungen zu, ${ }^{22}$ die die Konformität von Produkten, Verfahren, Dienstleistungen, Systemen mit europäischen und nationalen Normen und/oder Standards prüfen und damit die Möglichkeit des Marktzutritts bewirken. Die die Konformität prüfenden Stellen sind Private, die die Konformität für Private prüfen und damit deren Produkten und Dienstleistungen Zeichen oder Siegel verleihen. Dieses System ist europäisch weitgehend harmonisiert und wird in Deutschland durch das Gesetz über die Akkreditierungsstelle ${ }^{23}$ (AkkStelleG) geregelt, das der Umsetzung der VO EG Nr. 765/2008 dient und die Akkreditierung und Marktüberwachung in wichtigen Bereichen regelt. ${ }^{24}$ Als zentrale Stelle der Zulassung von Konformitätsbewertungen dient die Deutsche Akkreditierungsstelle GmbH (DAkkS), deren Geschäftszweck die Durchführung von Akkreditierungsaufgaben nach dem AkkStelleG ist und deren Gesellschafter zu gleichen Teilen der Bund, fünf Bundesländer sowie der Bundesverband der Deutschen Industrie e.V. (BDI) sind. Dazu ist die DAkkS nach $\mathbb{} 8$ AkkStelleG Beliehene. ${ }^{25}$ Sie nimmt also die Akkreditierung, wie in $\$ 1$ Abs. 1 AkkStelleG vorgesehen, als eine hoheitliche Aufgabe wahr. ${ }^{26}$ Sie teilt diese Aufgabe teilweise mit einigen anderen Stellen in anderen Bereichen, etwa dem der Deutschen Akkreditie-

19 Art. 2 Nr. 10 VO (EG) 765/2008.

20 Art. 2 Nr. 12 VO (EG) 765/2008.

21 Zum New Legislative Framework als gemeinschaftlichem Rahmen der Vermarktung von Produkten vgl. G. Dimitropoulos, Zertifizierung (Fn. 8), S. $91 \mathrm{ff}$.

22 Zum Teil wird die Akkreditierung auch von Behörden vorgenommen.

23 Akkreditierungsstellengesetz v. 31.7.2009 (BGBl I S. 2625).

24 Dies gilt allerdings nur insoweit, wie in anderen Vorschriften nichts abweichendes geregelt ist, etwa für Medizinprodukte, Gendiagnostika, Sicherheitstechnik sowie Ernährung, Landwirtschaft und Verbraucherschutz einschließlich der Lebensmittelsicherheit; vgl. $\$ 1$ Abs. 2 AkkStelleG.

25 Die Anforderungen ergeben sich aus Art. 8 VO EG 765/2008 i.V.m. $\$ 10$ AkkStelleG; zur Beleihung der DAkks vgl. Verordnung über die Beleihung der Akkreditierungsstelle nach dem Akkreditierungsstellengesetz v. 21.12.2009, BGBl I, S. 3962.

26 Insoweit besteht eine öffentlich-rechtliche Auffangfunktion für den Fall, dass eine private Stelle nicht beliehen wird oder diese Tätigkeit beendet; dann kann ein Bundesamt für Akkreditierung errichtet werden. Vgl. $\$ 8$ Abs. 2 AkkStelleG. 
rungs- und Zulassungsgesellschaft für Umweltgutachter (DAU) ${ }^{27}$ nach Maßgabe der EMAS-Verordnung. ${ }^{28}$

Die Akkreditierung als Konformitätsbewertungsstelle geschieht auf Antrag einer Einrichtung für einen bestimmten, klar definierten Bereich nach einem Verfahren, das den gemäß $\ 5$ Abs. 2 Nr. 1 u. 2 AkkStelleG vom Akkreditierungsbeirat ermittelten und gemäß $\$ 5$ Abs. 3 AkkStelleG vom Bundesministerium für Wirtschaft und Energie bekannt gemachten „Allgemeinen Regeln zur Akkreditierung von Konformitätsbewertungsstellen “29 entspricht. Dabei entscheidet ein Akkreditierungsausschuss, dessen (wechselnde) Zusammensetzungen fachbezogen für den jeweiligen Bereich ausgewählt sind. ${ }^{30}$

Die bei der Akkreditierungsstelle akkreditierten Stellen führen sodann die Konformitätsbewertungen durch. Die von der akkreditierten Stelle auszustellende Konformitätsbescheinigung bezieht sich auf die Einhaltung der für den jeweiligen Bewertungsgegenstand geltenden Regelungen und Normen. Insoweit handelt es sich verbreiteter Auffassung nach um eine private Bescheinigung von Rechts- und Standardkonformität und damit gleichsam um eine indirekte Durchsetzung des Rechts in einem durch öffentlich-rechtliche Normen aufgespannten System. ${ }^{31}$ Folglich ist die Beziehung zwischen der akkreditierten Konformitätsbewertungsstelle und dem Hersteller von Produkten daher auch eine privatrechtliche Rechtsbeziehung. ${ }^{32}$ Indes zeigt sich auch die charakteristische Schwierigkeit der Einordnung der Tätigkeit in das System des deutschen Verwaltungsrechts, ${ }^{33}$ dem private Formen des Vollzugs des öffentlichen Rechts und private Entscheidungen etwa als Marktzugangsvoraussetzungen lange Zeit fremd waren. Von daher fanden (und finden) sich vielfältige Versuche der Einordnung dieser Tätigkeiten als Beleihung, die damit öffentliche (oder

27 Vgl. dazu Verordnung über die Beleihung der Zulassungsstelle nach dem Umweltauditgesetz (UAGBeleihungsverordnung - UAGBV) v. 18. Dezember 1995 (BGBl. I S. 2013), zuletzt geändert durch Art. 1 der Verordnung vom 13. Dezember 2011 (BGBl. I 2727).

28 Inwieweit diese Zersplitterung mit der VO (EG) 765/2008 vereinbar ist, unterliegt durchaus Zweifeln, nicht zuletzt im Hinblick auf Art. 3 Nr. 30 VO (EG) 1221/2009. Vgl. J. Windmann, Der Verifikateur und der Aufsichtsbeamte als zentrale Elemente des Sachverständigen-Vollzugsmodells im Technikrecht, DÖV 2010, 396 (2398); G. Dimitropoulos, Zertifizierung(Fn. 8), S. 74.

29 Allgemeine Regelungen zur Akkreditierung von Konformitätsbewertungsstellen v. 29.8.2012, abrufbar unter http:/www.dakks.de/content/allgemeine-regeln-zur-akkreditierung-von-konformit $\%$ C3\%A4tsbewertungsstellen.

30 Vgl. $\$ 10$ Abs. 1 Nr. 3 AkkStelleG; dazu auch W. Tiede/ Ch. Ryczewski/ M. Yang, Akkreditierungsrecht (Fn. 8), S. 1212 (1214).

31 K. Bieback, Zertifizierung (Fn. 8), S. 226 ff.; J. Hofmann, Rechtsschutz und Haftung im europäischen Verwaltungsverbund, 2004, S. 28 f.; U. Kage, Das Medizinproduktegesetz, 2005, S. 197; Merten, Benannte Stellen, DVBl 2004, S. 1211 ff.; H. Pünder, Akkreditierung (Fn. 10), S. 578 ff.; E. SchmidtAßmann, Das Allgemeine Verwaltungsrecht als Ordnungsidee, Berlin/Heidelberg/New York 20043. Kap Rn. 57.

32 Ungeachtet sonstiger Fragen der Wirkung handeln die Konformitätsbewertungsstellen wie auch andere zertifizierende Einrichtungen im Auftrag der jeweiligen privaten Akteure, vgl. schon A. Seidel, Privater Sachverstand (Fn. 3), S. $269 \mathrm{ff}$.

33 Dazu E.Schmidt-Aßmann, Ordnungsidee (Fn. 31), 3. Kap. Rn. 57. 
staatliche) Aufgaben als ein Teil der öffentlichen Verwaltung wahrnehmen sollen ${ }^{34}$ oder aber als Repräsentanten einer ggf. europäisch institutionalisierten Hoheitsgewalt. ${ }^{35}$ Der Sache nach handelt es sich freilich um ein Mischsystem von öffentlichrechtlichen und privaten Elementen. ${ }^{36}$ Die Mischform ändert nichts daran, dass die Stellung der daran beteiligten Akteure jeweils nach Maßgabe der gesetzlichen Ausgestaltung ebenso zu bestimmen ist wie die konkrete Verantwortlichkeit innerhalb der Regelungsstruktur. Diese setzt sich im Übrigen im internationalen Bereich fort, in dem eine Zertifizierungsinfrastruktur entstanden ist, die der Durchsetzung internationaler, europäischer und nationaler Normen und Standards durch eine Konformitätsbewertung dient, die nicht in das hoheitliche Regime eingegliedert wird, wohl aber in einem durch die genannten Normen aufgespannten Rahmen handelt. ${ }^{37}$

\section{Zulassung von Trägern und Maßnahmen im Sozialrecht - ein bestrittenes Arrangement}

Die Schwierigkeiten in der rechtlichen Konstruktion zeigen sich exemplarisch im Sozialrecht. Danach bedürfen etwa Träger und auch bestimmte Maßnahmen der Zulassung durch eine fachkundige Stelle ( $\mathbb{S} 176$ SBG III). Fachkundige Stellen sind die von der Akkreditierungsstelle für die Zulassung nach dem Recht der Arbeitsförderung akkreditierten Zertifizierungsstellen ${ }^{38}$ wobei mit der Akkreditierung ausdrücklich keine Beleihung verbunden ist ( $\$ 177$ Abs. 1 S. 2 SGB III). Diese fachkundigen Stellen sind für die Zulassung von Trägern und Maßnahmen nach $\$ 177$ SGB III zuständig. Ungeachtet der Tatsache, dass der Gesetzestext mittlerweile feststellt, dass mit der Akkreditierung keine Beleihung verbunden ist und in der Gesetzesbegründung ausdrücklich festgehalten wird, dass die fachkundigen Stellen bei der Zulassung von Trägern und Maßnahmen wie bisher in den Formen des Privatrechts als unabhängige Sachverständige handeln, ${ }^{39}$ soll nach Auffassung des Bundessozialgerichts, das allerdings noch das alte Recht der $\mathbb{\$} \mathbb{8 4}, 85$ SGB III zum Gegenstand hatte, wohl aber zum seinerzeitig schon vorliegenden Gesetzesentwurf der $\mathbb{S} 176 \mathrm{ff}$. SGB III Stellung genommen hat ${ }^{40}$ die Entscheidung der fachkundigen Stellen einen Verwal-

34 K.-Ch. Scheel, „Benannte Stellen“: Beliehene als Instrument für die Verwirklichung des Binnenmarktes, DVBl. 1999, 442 ff.; St. Kadelbach, Allgemeines Verwaltungsrecht unter europäischen Einfluss, Tübingen 1999, S. $329 \mathrm{f}$ (differenzierend); F. Reimer, Qualitätssicherung, Grundlagen eines Dienstleistungsverwaltungsrechts, Baden-Baden 2010, S. $286 \mathrm{f}$.

35 Vgl. H.C. Röhl, Akkreditierung (Fn. 8), S. 28 ff.; ders., Konformitätsbewertung (Fn. 8), S. 153 (166 f.).

36 Insoweit zutreffend G. Dimitropoulos, Zertifizierung (Fn. 8), S. 126 ff., ohne das freilich die Begriffe „Regulierter Selbstregulierung“ oder "Re-Regulierung" als heuristische Beschreibungsformen schon etwas zur dogmatischen Lösung beizutragen vermögen.

37 Ausführlich G. Dimitropoulos, Zertifizierung (Fn. 8), S. $243 \mathrm{ff}$.

38 Akkreditierungsstelle ist seit dem 1.4.2012 die DAkkS nach Maßgabe des AkkStelleG; vgl. Neumann, in: Rohlfs/ Giesen/ Krekebohm/ Udsching, Beck'scher Online Kommentar Sozialrecht $\$ 177$ Rn. 2; M. Banafsche (Fn. 11), SGB III $\$ 176$ Rn. 4 ff.

39 BT-Drucks. $17 / 6277$ S. 107 zu $\$ 177$.

40 BSG, Beschluss vom 3.8.2011 - B 11 SF 1/10 R - Rn. 28. 
tungsakt darstellen, der dann entsprechend auch vor den Sozialgerichten anzufechten ist. ${ }^{41}$ Dies wird nicht zuletzt damit begründet, dass es eine enge Verzahnung der maßnahmenbezogenen Fördervoraussetzungen mit dem Zertifizierungsverfahren und den Voraussetzungen der Fördermaßnahmen gibt. Da es den Erlass hoheitlicher Maßnahmen ohne eine gesetzliche Beleihung nicht geben kann, das Gesetz eine Beleihung aber ausschließt, ${ }^{42}$ ist diese Auffassung obsolet geworden. Um den Sachzusammenhang zu den Regelungen des Sozialrechts und damit die Zuständigkeit der Sozialgerichte aufrechtzuerhalten reichen pragmatische Erwägungen nicht aus, zumal mit dem Wechsel zu der Akkreditierungsstelle auch auf das eindeutig anders strukturierte Modell des Akkreditierungsstellengesetzes und der dazu ergangenen Rechtsverordnungen Bezug genommen wird, also das zunächst im Produktsicherheitsrecht entwickelte Modell ausdrücklich aufgenommen wird. Allerdings spricht das in einem Teil der sozialrechtlichen Literatur erkennbare Unbehagen an dieser gesetzlichen Lösung für sich.

\section{Das System der Akkreditierung im Hochschulbereich}

Vor diesem Hintergrund lässt sich das System der Akkreditierung auch im Hochschulbereich profilieren. Seine Strukturelemente mögen für den Hochschulbereich seinerzeit neu gewesen sein, insgesamt aber bedient sich auch dieses System durchaus der bekannten strukturellen Elemente, wenn auch mit einer Anpassung an die Besonderheiten des Hochschulsektors. Dies zu betonen ist nicht unwichtig; denn in manchen Stellungnahmen erscheint es so, als sei diese Form weitgehend unabhängig von anderen Bereichen entstanden. Demgegenüber ist diese Teil einer Neustrukturierung des Qualitätssicherungsbereichs und insofern nicht unabhängig von der Entwicklung in diesen Bereichen entstanden. ${ }^{43}$ Die institutionelle Ausformung ist dabei

41 Vgl. M. Banafsche (Fn. 11), SGB III $\ 176$ R. 40 ff. m.w.N, wo ausführlich die bisherige Rechtsauffassung entfaltet wird. Zum zentralen Argument wird dabei, dass es sich um die Vollziehung öffentlichrechtlicher Normen und daher nach wie vor um eine als Verwaltungsakt besser zu qualifizierende Maßnahme handele. Indes wird dabei übersehen, dass die Normen über die Zulassung eben gerade keinen Träger öffentlicher Gewalt verpflichten. Davon abgesehen, ist die Struktur von vornherein eben als eine gemischte etabliert worden. Zum Ganzen auch Mutschler/ Schmidt-DeCaluwe/ Coseriu/ Scholz, SGB III 5. Aufl. 2013, 176 Rn. 29 ff.; A. Hänlein, Steuernder Akteur im Wirtschaftsleben, Soziale Sicherheit 2005, 273 (274); ders., Förderung der beruflichen Weiterbildung - Zulassung von Weiterbildungsträgern und -maßnahmen, SGb 2011, 333 (335 f.). Ähnliche Probleme zeigen sich im Sozialrecht bei den sog. Präqualifizierungsstellen vgl. dazu E. Zimmermann, Erfahrungen mit dem neuen Präqualifizierungsverfahren im SGB V, NZS 2013, S. 463 ff.; R. Flasbarth, Präqualifizierungsprobleme nach $\mathbb{S} 126$ a Abs. 1 SGB V - Rechtsnatur, Verfahren, Probleme, MedR 2011, S. 77 ff.

$42 \int 177$ Abs. 1 E-SGB II enthielt zum Zeitpunkt der Entscheidung des BSG (Fn. 40) den entsprechenden Ausschluss der Beleihung nicht. Vielmehr ist dies als ausdrückliche Klarstellung mit Bezug auf die Entscheidung des BSG durch das Gesetz zur Neuordnung der Altersversorgung der Bezirksschornsteinfeger und zur Änderung anderer Gesetze eingeführt worden; vgl. BT Drucks. 17/10749 S. 17. Zugleich ist durch Art. 5 des Gesetzes eine Änderung des $\$ 51$ Abs. 2 SGG vorgenommen worden, der im Hinblick auf diese Rechtsprechung die Zuständigkeit der Sozialgerichte und ihre gewonnenen Erfahrungen erhält; vgl. BT Drucks 17/10749 S. 17.

43 Darauf verweist zu Recht Wissenschaftsrat, Empfehlungen (Fn. 12), S. 44 f., ebenso wie auf die Notwendigkeit einer Adaption der Konzepte. 
Teil eines kontinuierlichen Lernprozesses, der von Pilotprojekten über Evaluation und ggf. die Revision des Gesamtsystems der Akkreditierung reicht. ${ }^{44}$ Sie ist eingebettet in die allgemeine Veränderung des Verhältnisses von Staat und Hochschule. ${ }^{45}$ Diese zeigt sich in der gewollten Stärkung der Unabhängigkeit der Hochschulen, ihrer Strategiefähigkeit, die Voraussetzung für eine Profilbildung im Wettbewerb ist. Diese soll sich nunmehr auch auf die Lehre und damit die Angebote an Studiengängen erstrecken. Auch eine verstärkte Internationalisierung der Angebote legte andere Formen der Qualitätssicherung nahe, die nicht mehr an den früheren, eher statischen, an quantitativen Vorgaben orientierten Anforderungen mit staatlicher Genehmigung ausgerichtet waren. Vielmehr bedurfte es differenzierter und dynamischerer Modelle, die die Anforderungen inhaltlich nicht mehr auf ein für alle gleichermaßen geltendes und ausgerichtetes Angebot ausrichten, gleichwohl aber bestimmte Qualitätsstandards setzen. Zugleich sollte die Verantwortung für die Qualitätssicherung stärker in die scientific community verlagern werden. ${ }^{46}$ In einem staatlich gesetzten Rahmen sollte damit eine kontinuierliche Orientierung auch an der europäischen Weiterentwicklung der Qualitätsstandards ermöglicht werden. Sie ist daher eingebettet in einen europäischen Rahmen, der darauf zielt, einen europäischen Hochschulraum auch und gerade für die Studierenden zu schaffen. ${ }^{47}$ Eine zentrale Rolle spielen die „European Standards and Guidelines for Quality Assurance in the European Higher Education Area“, die von der Ministerkonferenz in Bergen beschlossen worden sind. ${ }^{48}$ Auf europäischer Ebene ist zudem im Zuge der Herausbildung nationaler Akkreditierungssysteme die European Association for Quality Assurance in Higher Education (ENQA) entstanden, die sich aus Akkreditierungsagenturen der Mitgliedstaaten der Europäischen Union zusammensetzt und ein horizontales Netzwerk nationaler Agenturen bildet, das diese mit europäischen Akteuren verbindet. Darüber hinaus ist die externe Qualitätssicherung der Hochschullehre partiell auch in das

44 Der Prozess und die je unterschiedlichen Interessen und Positionen werden nachgezeichnet bei $U$. Banscherus, Qualitätssicherung in Studium und Lehre in der hochschulpolitischen Auseinandersetzung, Bericht im Auftrag der Max-Traeger-Stiftung, Frankfurt/M. 2011, S. 31 ff., 43 ff.; Wissenschaftsrat, Empfehlungen (Fn. 12), S. 16 ff.; U. Erichsen, Drei Jahre Qualitätssicherung durch Akkreditierung - Eine Zwischenbilanz, Die Hochschule 12 (2003), $160 \mathrm{ff}$.

45 Ausführlich dazu H.-H. Trute/ A. Pilniok, in: Jansen (Hrsg.), Neue Governance für die Forschung, Baden-Baden, 2009, S. 21 ff.; B. Kaufmann, Akkreditierung als Mikropolitik. Zur Wirkung neuer Steuerungsinstrumente an deutschen Hochschulen, Wiesbaden 2012; S. Sieweke, Managementstrukturen und output-orientierte Finanzierung im Hochschulbereich, Baden-Baden 2010.

46 U. Erichsen (Fn. 44), Zwischenbilanz, S. 161.

47 Auf der Berliner Ministerkonferenz (vgl. dazu http://www.bologna-berlin2003.de) ist die Rolle der Qualitätssicherung zur Verwirklichung des Europäischen Hochschulraums hervorgehoben worden. Siehe http://www.bologna-berlin2003.de/pdf/Communique1.pdf. Zum europäischen Hochschulraum vgl. Amaral et al. (eds.), European Integration and the Governance of Higher Education and Research.

48 Siehe http://www.bmbf.de/pubRD/bergen_kommunique_dt.pdf; zu deren Rolle auch Wissenschaftsrat, Empfehlungen (Fn. 12), S. 66 ff.,76. 
Unionsrecht eingebettet. ${ }^{49}$ Dies lässt sich als Umstellung von einer statischen Qualitätssicherung durch Rahmenprüfungsordnungen hin zu einem peer-review ähnlichen Verfahren für die Bologna-Struktur der Studiengänge ${ }^{50}$ im Rahmen einer staatlichen Rahmenverantwortung verstehen. Diese aus Pilotprojekten hervorgegangene Entwicklung ist noch nicht abgeschlossen. Sie zielt derzeit auf eine Zertifizierung von Qualitätssicherungssystemen, die eine auf den einzelnen Studiengang bezogene externe Qualitätsprüfung überflüssig machen soll..$^{51}$

\section{Staatliche Verantwortung - intermediäre Strukturen - Eigenverantwortung}

Entstanden ist auf diese Weise in mehreren Schritten eine durchaus komplexe Regelungsstruktur. Von der Anlage her sollen in einem staatlich aufgespannten Rahmen unter staatlicher Aufsicht die staatlichen Regeln und Standards konkretisiert und umgesetzt werden und die fachlich-inhaltliche Qualität zudem nach Maßgabe des aus dem Bereich der Forschung wohlbekannten Verfahrenselement des peer review bewertet werden. Dies geht einher mit der Institutionalisierung einer Entwicklungsfunktion für die Qualitätssicherung, die in den oben erwähnten europäischen Rahmen eingebettet ist. Schon die Einführung der Systemakkreditierung ist ein Beispiel für die Entwicklungsfunktion, ebenso die Reaktionen auf die zu starke Detaillierung, die eine erneute Anpassung der Voraussetzung der Akkreditierung zur Folge hatte. ${ }^{52}$ Der Sache nach wird damit die vorherige zweistellige Beziehung von Staat und Hochschulen ${ }^{53}$ mit der staatlichen Genehmigung von Studiengängen durch eine mehrstellige Relation ersetzt, indem zwischen beide Pole das System der Akkreditierung tritt, mit je unterschiedlichem Verhältnis zu etwaige erforderlichen Genehmigungen.

49 Empfehlung des Rates vom 24. September 1998 betreffend die europäische Zusammenarbeit zur Qualitätssicherung in der Hochschulbildung (98/516/EG), ABl. 1998 L 270/56; Empfehlung des Europäischen Parlamentes und des Rates vom 15. Februar 2006 über die verstärkte europäische Zusammenarbeit zur Qualitätssicherung in der Hochschulbildung (2006/143/EG), ABl. 2006 L 64/60.

50 Zu Beginn des Wintersemesters 2012/2013 waren rund 87 \% der Studiengänge auf die gestufte Studienstruktur ausgerichtet, der Großteil der übrigen Studiengänge führe zu kirchlichen und staatlichen Abschlüssen; vgl. http://www.bmbf.de/de/3336.php.

51 Vgl. dazu Wissenschaftsrat, Empfehlungen (Fn. 12), S. 84ff.; Die Hochschulrektorenkonferenz plädiert für eine von externen Bewertungen unabhängiges Audit; dazu das von vbw- Vereinigung der Bayerischen Wirtschaft e.V. herausgegebene Gutachten von H.P. Blossfeld/ W. Bos/ H.-D.Daniel/ B.Hannover/ D. Lenzen/ M. Prenzel/ H.-G. Roßbach/ R. Tippelt/L. Wößmann, Qualitätssicherung an Hochschulen: von der Akkreditierung zur Auditierung, Münster 2013.

52 Vgl. Wissenschaftsrat, Empfehlungen (Fn. 12), S. $32 \mathrm{ff}$.

53 Die freilich in der Sache immer schon etwas komplizierter war, nicht zuletzt durch die Einbettung in die föderalen Abstimmungsmechanismen und die Generierung von Rahmenprüfungsordnungen im Zusammenspiel von KMK, HRK und Berufspraxis und der Transformation in örtliche Ordnungen, die dann ministeriell genehmigt wurden. Vgl. dazu die Schilderung der KMK http://www.kmk.org/ wissenschaft-hochschule/studium-und-pruefung/rahmenpruefungsordnungen.html.; KMK, Künftige Entwicklung der länder- und hochschulübergreifenden Qualitätssicherung in Deutschland, Beschluss v. 1.3.2002. 


\section{Staatliche Verantwortung für die Qualitätssicherung}

Dabei verbleibt die staatliche Verantwortung für die Qualität des Hochschulsystems von der Einrichtung des Systems der Akkreditierung durchaus unberührt. Diese staatliche Verantwortung lässt sich jedenfalls auch aus der objektiv-rechtlichen Dimension der Wissenschaftsfreiheitsgarantie des Art. 5 Abs. 3 GG, hier in Gestalt der Lehrfreiheit, begründen. Das Bundesverfassungsgericht hat auch in jüngeren Entscheidungen an der Formulierung einer Gewährleistungsverantwortung des Gesetzgebers festgehalten, wonach der Staat, der sich als Kulturstaat verstehe, verpflichtet sei, funktionsfähige Einrichtungen für einen freien Wissenschaftsbetrieb zur Verfügung zu stellen. ${ }^{54}$ Der Staat hat durch geeignete organisatorische Maßnahmen sicherzustellen, dass das individuelle Grundrecht der freien wissenschaftlichen Betätigung soweit unangetastet bleibt, wie das unter Berücksichtigung der anderen legitimen Aufgaben der Wissenschaftseinrichtungen und der Grundrechte der verschiedenen Beteiligten möglich ist. ${ }^{55}$ Dies kann man - weniger kulturstaatlich ausgerichtet - die Gewährleistungsverantwortung des Staates für die freie Wissenschaft nennen. ${ }^{56}$ Dies umschließt auch die Verantwortung für die Qualitätssicherung, die für andere Grundrechtsträger, etwa die Studierenden von erheblicher Bedeutung ist, wie es ein Interesse der Gesellschaft daran gibt, funktionierende Einrichtungen der Lehre zu haben. Insoweit umschließt die Gewährleistungsverantwortung des Staates für funktionierende Einrichtungen der Wissenschaft auch die Verantwortung für eine Qualitätssicherung der Lehre. Ein ähnliches Ergebnis könnte man aus Perspektive der Rechte der Studierenden erzielen. Folgerichtig heißt es in der Begründung zum ASG, dass die staatliche Verantwortung für die Qualität des Hochschulsystems unberührt bleibe. ${ }^{57}$ Freilich umschließt die staatliche Verantwortung unterschiedliche Dimensionen. Neben der Vorgabe bestimmter Qualitätsziele beinhaltet diese vor allem eine institutionelle und prozedurale Verantwortung.

\section{Eigenverantwortung der Hochschulen für die Qualitätssicherung}

Sie findet allerdings zugleich eine Grenze an der Eigenverantwortung der Hochschulen für die Qualitätsentwicklung und -sicherung. Qualitätssicherung ist insofern nicht nur Aufgabe des Staates. Das hat das Bundesverfassungsgericht an anderer Stelle ebenfalls so gesehen. „Aus der Schlüsselfunktion der freien Wissenschaft für die gesamtgesellschaftliche Entwicklung folgt auch eine Verantwortung für die Sicherung ihrer Leistungsfähigkeit. Die besondere Stellung der Hochschullehrer im Bereich der Universitäten und Fachhochschulen ist nur dann gewährleistet, wenn deren sachgerechte und allein an qualitativ-wissenschaftlichen Maßstäben ausgerichtete Auswahl gewährleistet wird. Mit der Prüfungsentscheidung über die Habi-

54 Std. Rspr. BVerfGE 35, 79 (115); 111, 333 (353); 127, 87 (114).

55 BVerfGE 127, 87 (114); 111, 333 (353); std. Rspr. vgl. bereits BVerfGE 35, 79 (115).

56 Vgl. allgemein D.Krausnick, Staat und Hochschule im Gewährleistungsstaat, Tübingen 2012.

57 Vgl. die Begründung zum ASG NW LT-Drucks 13/6182 S. 9. 
litation ist demgemäß auch eine institutionelle Verantwortung für die Qualitätssicherung der freien Wissenschaft (Herv. HHT) verbunden. " ${ }^{58}$ Auch insoweit folgt aus der Wissenschaftsfreiheitsgarantie eine institutionelle Verantwortung für die Qualitätssicherung, die je nach Kompetenzbereich der jeweiligen Einrichtung aktualisiert wird. Insofern obliegt den Hochschulen eine auch die Lehre treffende Verantwortung für hinreichende Formen der eigenen Sicherung der Qualität. Je mehr im Übrigen die Autonomie der Hochschulen in der Gestaltung der Lehre reicht, desto weiter wird man auch die Eigenverantwortung ziehen müssen. Dafür spricht, nicht anders als im Bereich der Forschung, in dem dies bereits deutlicher zu beobachten ist, dass mit der Betonung der Autonomie der Einrichtungen, die Gewährleistungsverantwortung nicht mehr ausschließlich durch den Gesetzgeber wahrgenommen werden kann, sondern eher einer prozeduralen Vorstrukturierung bedarf, die die Eigenverantwortung der Einrichtungen akzentuiert und Prozesse ihrer Anreizung und Wahrnehmung vorsieht. 59

\section{Die Zuordnung durch den Gesetzgeber}

Insoweit sind staatliche Verantwortung und Eigenverantwortung zuzuordnen. Dies dürfte eine Aufgabe des Gesetzgebers sein. Insoweit ist die Aussage des Bundesverfassungsgerichts richtig, dass der Gesetzgeber frei ist, den Wissenschaftsbetrieb nach seinem Ermessen zu regeln, um die unterschiedlichen Aufgaben der Wissenschaftseinrichtungen und die Interessen aller daran Beteiligten in Wahrnehmung seiner gesamtgesellschaftlichen Verantwortung in angemessenen Ausgleich zu bringen. ${ }^{60}$ Für diese Aufgabe ist, so das Bundesverfassungsgericht, der parlamentarische Gesetzgeber besser geeignet, als die an speziellen Interessen orientierten Träger der Wissenschaftsfreiheit. Ihm stehen dabei gerade hinsichtlich der Eignung neuer Organisationsformen eine Einschätzungsprärogative und ein Prognosespielraum zu. ${ }^{61}$ Dies aber bedeutet nicht, dass der Gesetzgeber eine staatliche Struktur vorsehen muss. Der Wissenschaftsbereich ist in der Forschung wie in der Lehre vielmehr durch eine Vielzahl von hybriden Arrangements gekennzeichnet, die die je unterschiedlichen Interessen an der Forschung und Lehre austarieren sollen. ${ }^{62}$ Gerade im Hinblick auf die weitergehende Autonomie der Einrichtungen und damit die notwendig damit einhergehende Differenzierung der Angebote, können Formen der eigenständigen Qualitätssicherung vorgesehen werden, die dann auch durch intermediäre Einrichtungen der Akkreditierung begleitet werden. Insofern käme es vor allem darauf an, dass die Ausgestaltung der damit einhergehenden institutionellen Struktur wissenschaftsad-

58 BVerfG, NVwZ 2011, $486 \mathrm{ff}$.

59 Zur prozeduralen Vorstrukturierung auch K.-H. Ladeur, Die Wissenschaftsfreiheit der entfesselten Hochschule, DÖV 2005, S. $753 \mathrm{ff}$.

60 Vgl. BVerfGE 35, 79 (116, 120); 47, 327 (404); 93, 85 (95); 111, 333 (353); 127, 87 (116).

61 Vgl. BVerfGE 50, 290 (332 f.); 88, 203 (262); 111, 333 (355 f.).

62 Ausführlich dazu H.-H. Trute, Ungleichzeitigkeiten in der Dogmatik: Das Wissenschaftsrecht, DV 27 (1994), S. $301 \mathrm{ff}$. 
äquat unter Berücksichtigung der übrigen Interessen an einer funktionierenden Wissenschaft vorgenommen wird. Insoweit kommt es auf die Ausgestaltung der institutionellen Struktur im Einzelnen an. Jedenfalls ist nichts grundsätzlich dagegen einzuwenden, ${ }^{63}$ dass die Länder ungeachtet der Verantwortung für die Qualitätssicherung die Wahrnehmung ibrer Aufgaben nach $\mathbb{9}$ Abs. 2 HRG im Wege einer institutionellen Beteiligungsverwaltung auf die Stiftung zur Akkreditierung von Studiengängen übertragen. ${ }^{64}$

\section{Staatliche Rahmenvorgaben}

Schon innerhalb des deutschen Föderalismus ist die Herstellung vergleichbarer Studiengänge und Abschlüsse nicht einfach. Dazu diente im Rahmen des kooperativen Föderalismus zunächst die frühere Rahmengesetzgebungskompetenz des Bundes, von der im vorliegenden Kontext der $\$ 9$ Abs. 2 HRG fortbesteht. Hier übernimmt vor allem die Kultusministerkonferenz die wichtige Rolle der Harmonisierung der Anforderungen. Dies galt schon unter dem Regime der Rahmenprüfungsordnungen, gilt aber natürlich auch im Hinblick auf die Anforderungen an die Bachelor- und Masterstudiengänge.

So muss die notwendige Vergleichbarkeit der Bildungsabschlüsse über die gesetzlichen und von der KMK beschlossenen ländergemeinsamen Strukturvorgaben ${ }^{65}$ gesichert werden, die von dem Akkreditierungsrat gemäß $\$ 2$ Abs. 1 Nr. 2 ASG zusammen mit den landesspezifischen, der Absicherung der landespolitischen Zielvorgaben dienenden Strukturvorgaben als verbindliche Vorgaben für die Agenturen bei der Akkreditierung zusammenzufassen sind. Die Strukturvorgaben beziehen sich auf die allgemeinen Festlegungen zur Regelstudienzeit ohne Fach- oder Abschlussdifferenzierungen sowie Vorgaben zur Studienstruktur, zu Zugangsvoraussetzungen und -übergängen, zur Ausrichtung des jeweiligen Studiengangs und zur Wertigkeit der Abschlüsse im Vergleich zum herkömmlichen Graduierungssystem. Darüber hinaus sollen die Standards der peers, die sich aus dem fachlichen Diskurs der jeweiligen

63 Die Frage, ob dies eines Staatsvertrages bedarf, oder - wie geschehen - in Form eines Verwaltungsabkommens dergestalt geschehen kann, dass eine Einrichtung nach dem Recht eines Landes gegründet und qua Verwaltungsabkommen von allen in Anspruch genommen wird, ist eine hier nicht näher zu behandelnde Frage. Die Regelung durch Verwaltungsabkommen entspricht einer nicht nur im Wissenschaftsbereich lang gepflegten Tradition, die angesichts weitreichender Wirkungen durchaus zweifelhaft ist; dazu D. Immer, Rechtsgrundlagen (Fn. 10), S. 359 ff.; für Art. 91b GG kritisch H.-H. Trute, Verwaltungskompetenzen nach der Föderalismusreform - zwischen Entflechtung und Verflechtung, in: F. Hufen (Hrsg.), Verfassungen - zwischen Recht und Politik, FS H.-P. Schneider, BadenBaden 2008, S. 302 (317ff.); zurückhaltender etwa O. Jauch, Wissenschaftsförderung nach der Reform des Föderalismus, Diss. Hamburg 2013, S. 89 ff., 213 ff. jeweils mit w. Nachw.

64 NW LT-Drucks 13/6182 S. 9 f. Dazu Vereinbarung der KMK zur Stiftung „Stiftung: Akkreditierung von Studiengängen in Deutschland“, abrufbar unter http:/www.kmk.org/fileadmin/veroeffentlichungen_beschluesse/2004/2004_12_16-Vereinbarung-Akkreditierung-Studiengaenge.pdf.

65 Ländergemeinsame Strukturvorgaben für die Akkreditierung von Bachelor- und Masterstudiengängen, Beschluss der Kultusministerkonferenz vom 10.10.2003 i.d.F. vom 4.04.2010, abrufbar unter http://www.kmk.org/fileadmin/veroeffentlichungen_beschluesse/2003/2003_10_10-Laendergemeinsame-Strukturvorgaben.pdf. 
scientific community hinsichtlich der inhaltlichen Anforderungen an eine berufsqualifizierende Hochschulausbildung in dem jeweiligen Fachgebiet ergeben, für das Akkreditierungsverfahren zugrunde gelegt werden. Insoweit handelt es sich um ein gemischtes System von Anforderungen, die für den Wissenschaftsbereich nicht untypisch sind: ein Zusammenspiel von staatlichen Vorgaben und fachlich-wissenschaftlichen Standards, die dann innerhalb der Regelungsstruktur durch geeignete Verfahren zur Geltung gebracht werden sollen.

Für die Kriterien und Verfahren gelten die Regeln, die den Rahmen für die Akkreditierungsagenturen umschreiben und die vom Akkreditierungsrat nach Maßgabe des $\ 2$ Abs. 1 Nr. 3 ASG beschlossen werden. ${ }^{66}$ Von der Konzeption her folgerichtig beschränkt sich die Aufgabe des Akkreditierungsrates auf die Regelung von Mindestvoraussetzungen für Akkreditierungsverfahren, um nicht die Hochschulen und die Agenturen durch die Vorgaben von Regeln unverhältnismäßig einzuschränken. Dies hängt nicht zuletzt damit zusammen, dass hinreichender Raum für die Entwicklungsfunktion des Gesamtsystems bleiben soll. ${ }^{67}$

\section{Die institutionelle Struktur der Akkreditierung}

Die Regelungsstruktur des intermediären Akkreditierungsbereiches lässt sich einerseits über das Verhältnis von Staat und der mittlerweile gesetzlich gegründeten zentralen Akkreditierungseinrichtung beschreiben. Diese setzt sich fort im Verhältnis zwischen der Akkreditierungseinrichtung und den bewertenden Stellen und wird drittens über das Verhältnis von bewertenden Stellen und zu bewertende Objekte beschrieben. Damit tritt, wie oben dargelegt, zwischen die staatlichen Hochschuladministrationen und die Hochschulen eine intermediäre Struktur, bei der der Akkreditierungsrat stärker für die staatlichen Steuerungsinteressen und die Agenturen stärker für das wissenschaftsorientierte peer review-Verfahren zuständig sind.$^{68} \mathrm{Dar}$ überhinaus ist das Verhältnis von Hochschulen und ihren Trägern nach Maßgabe des jeweiligen Landesrechts in Bezug auf die Akkreditierung in den Blick zu nehmen.

66 Vgl. Akkreditierungsrat, Regeln für die Akkreditierung von Studiengängen und für die Systemakkreditierung, Beschluss v. 8.12.2009, zuletzt geändert 20.2.2013, Drs. AR 20/2013.

$67 \mathrm{Ob}$ dies immer gelingt oder der Akkreditierungsrat nicht zu sehr in die Rolle einer bürokratischen Regulierungsinstanz hineingewachsen ist, mag zweifelhaft sein. Vgl. dazu Wissenschaftsrat, Empfehlungen (Fn. 12), S. 83ff.

68 Die Anforderungen an Intermediäre sind vor allem organisationsbezogen entwickelt worden; vgl. dazu H.-H. Trute, Legitimation (Fn. 7), Rn. 93 ff.; ders., Funktionen der Organisation und ihre Abbildung im Recht, in: E. Schmidt-Aßmann/W. Hoffmann-Riem (Hrsg.), Verwaltungsorganisationsrecht als Steuerungsressource, Baden-Baden 1997, S. 249 (266 ff.); E. Schmidt-Aßmann, Ordnungsidee (Fn. 7), 5. Kap. Rn. 59. Insoweit sind intermediäre Organisationen, die an der Schnittstelle von Staat und anderen gesellschaftlichen Bereichen die Funktion der Vermittlung von staatlichen Orientierungen und solchen des jeweiligen Sachbereichs haben, wie etwa die Deutsche Forschungsgemeinschaft und Mittlerorganisationen. Es spricht allerdings nichts dagegen, dass diese Funktionen der Vermittlung auf unterschiedliche Organisationen in einer Regelungsstruktur verteilt werden, wie dies in der Akkreditierung geschieht. Vielmehr kann dies zur deutlicheren Trennung unterschiedlicher Funktionen und damit zur Transparenz beitragen. 


\section{Der Akkreditierungsrat als Organ der "Stiftung zu Akkreditierung von Studiengängen in Deutschland"}

Die Schaffung des Akkreditierungsrates als zunächst unselbständige Einrichtung, ${ }^{69}$ die bei der Hochschulrektorenkonferenz und der Kultusministerkonferenz angesiedelt war, ${ }^{70}$ entsprach seinerzeit durchaus den Vorstellungen und Forderungen der Hochschulrektorenkonferenz. Der Akkreditierungsrat sollte danach die Funktion einer zentralen, die Akkreditierung von Studiengängen selbst vornehmenden Einrichtung haben. ${ }^{71}$ Stattdessen setzten die Kultusminister auf einen Wettbewerb verschiedener, noch zu gründender Agenturen. ${ }^{72}$ Diese zunächst nur experimentell eingerichtete Struktur ist 2005 auch als Reaktion auf Kritik an der mangelnden rechtlichen Ausformung durch ein Gesetz des Landes Nordrhein-Westfalen in die „Stiftung zur Akkreditierung von Studiengängen in Deutschland" überführt worden. ${ }^{73}$ Wie oben dargelegt, haben die Bundesländer der Stiftung durch Verwaltungsabkommen die Aufgaben des seinerzeitigen Akkreditierungsrates sowie die Wahrnehmung ihrer Aufgaben im Vollzug der ländergemeinsamen Strukturvorgaben für die Akkreditierung gemäß $\$ 9$ Abs. 2 HRG übertragen. ${ }^{74}$

\section{a. Die Funktion und Zusammensetzung des Akkreditierungsrates}

Die Stiftung, insbesondere der Akkreditierungsrat, nimmt eine Vermittlungsfunktion wahr, bei der aber innerhalb der Regelungsstruktur die staatliche Funktion im Vordergrund steht, diese aber mit der Beteiligung unterschiedlicher stakeholder ausgeübt werden sollen. Insoweit ist er auf der einen Seite zentraler Knoten in dem Netzwerk der Akkreditierung, zum anderen auch eine intermediäre Einrichtung. ${ }^{75}$ Sie vermittelt einerseits die staatliche Verantwortung für die Qualitätssicherung, ${ }^{76}$ anderseits ist sie, wie die Zusammensetzung der Organe zeigt, eine hochschulnahe Einrichtung. Darin ist sie nicht untypisch für höherstufige Einrichtungen im deutschen Wissenschaftssystem. ${ }^{77}$ Der Akkreditierungsrat, der über alle Angelegenheiten der Stiftung

69 Dazu U. Erichsen, Zwischenbilanz (Fn. 44), S. 160ff.; Wissenschaftsrat, Empfehlungen (Fn. 12), S. 20 ff.; K. Wilhelm, Verfassungs- und verwaltungsrechtliche Fragen (Fn. 10), S. 86 ff.; K.Bieback, Zertifizierung (Fn. 8), S. $132 \mathrm{ff}$.

70 Detailliert zu den Hintergründen K. Serrano-Vallarde, Evaluation (Fn. 10), S. 126 f.

71 Vgl. HRK, Akkreditierungsverfahren, Entschließung v. 6. Juli 1998; Wissenschaftsrat, Empfehlungen (Fn. 12), S. $20 \mathrm{ff}$.

72 Insoweit handelt es sich um ein für den Bildungsbereich durchaus neues, aber an den schon eingeführten Strukturen von Qualitätssicherungen in anderen Bereichen ausgerichtetes Modell.

73 Gesetz zur Errichtung einer Stiftung „Stiftung zur Akkreditierung von Studiengängen in Deutschland“ vom 15. Februar 2005, GV. Nordrhein-Westfalen, S. 45, zuletzt geändert durch das Gesetz zur Neuregelung des Kunsthochschulrechts vom 13. März 2008, GV. Nordrhein-Westfalen, S. 195 (im Folgenden: ASG).

74 Vgl. dazu oben C.) I.) 2.).

$75 \mathrm{Zu}$ intermediären Einrichtungen vgl. oben Fn. 68.

76 Vgl. dazu oben C.) II.).

77 Dazu H.-H. Trute, Ungleichzeitigkeiten (Fn. 61), S. 301 (318 ff.). 
beschließt, ${ }^{78}$ besteht aus 18 Mitgliedern, die von der Kultusministerkonferenz und der Hochschulrektorenkonferenz gemeinsam für vier Jahre bestellt werden. ${ }^{79}$ Aufsichtsorgan ist der Stiftungsrat, der sich aus sechs Vertretern der Kultusministerkonferenz und fünf Vertretern der Hochschulrektorenkonferenz zusammensetzt und die Arbeit des Akkreditierungsrates und des Vorstandes ${ }^{80}$ überwachen soll. ${ }^{81}$ Die Stiftung selbst unterliegt der Rechtsaufsicht des Ministeriums für Wissenschaft und Forschung NW.

\section{b. Die Aufgaben des Akkreditierungsrates}

Der Akkreditierungsrat soll zum einen als ein Forum der Diskussion und Anregung über die zukünftige Entwicklung der Qualitätssicherung wirken. ${ }^{82}$ Dies beinhaltet auch die Beobachtung der Qualitätsentwicklung in Europa und die Festlegung von Bedingungen der Anerkennung ausländischer Akkreditierungen. Er übernimmt zudem die bereits erwähnte Konkretisierungsfunktion für die Rahmenbedingungen der Akkreditierung in Umsetzung staatlicher Vorgaben und darüber hinaus die Akkreditierung und Re-Akkreditierung von Agenturen sowie die Überwachung der Akkreditierungen durch die Agenturen. Er unterliegt, nicht untypisch für höherstufige Einrichtungen des Wissenschaftssystems, der Evaluation, die ihrerseits dem Schema von Selbstreport, Begehung und Bewertung erfolgt. ${ }^{83}$

Insbesondere die Entwicklungsfunktion verlangt eine kontinuierliche Beobachtung des Akkreditierungsgeschehens, eine von dem Akkreditierungsrat offenbar nur begrenzt wahrgenommene, aber für die Weiterentwicklung des Systems wichtige Aufgabe. ${ }^{84}$ Damit werden zudem Informationen generiert, die für die Wahrnehmung der staatlichen Verantwortung bedeutsam sind und ggf. zu einer Neujustierung führen können.

78 \$ 7 Abs. 1 S. 1 ASG.

$79 \$ 7$ Abs. 2 ASG. Danach benennt die HRK vier Vertreter der Hochschulen und zwei Vertreter der Studierenden, die KMK vier Vertreter der Länder sowie einen Vertreter der für das Dienst- und Tarifrecht zuständigen Landesministerien, beide gemeinsam benennen vier Vertreter der beruflichen Praxis, zwei ausländische Vertreter mit Akkreditierungserfahrung sowie einen Vertreter der Agenturen mit beratender Stimme.

80 Dem Vorstand gehören der Vorsitzende und stellvertretende Vorsitzende des Akkreditierungsrates sowie der Geschäftsführer an; vgl. $\$ 8$ Abs. 2 ASG.

81 \$6 Abs. 1 Nr. 3, $\$ 9$ ASG.

82 Vgl. $\$ 11$ der Satzung der Stiftung zur Akkreditierung von Studiengängen in Deutschland v. 26. Juni 2006 (MBl. NRW. 2006 S. 431) zuletzt geändert am 20.2.2012 (MBl.NRW.2012 S. 164); zuletzt Report on the External Evaluation of the German Accreditation Council (Akkreditierungsrat) v. 31. Juli 2013 (abrufbar unter http://www.akkreditierungsrat.de/fileadmin/Seiteninhalte/AR/Sonstige/Gutachten_Evaluation_AR_2013_en.pdf.), S. 3.

83 Vgl. Report on the External Evaluation of the German Accreditation Council (Akkreditierungsrat) v. 31. Juli 2013. Überprüft wird die Übereinstimmung mit den gesetzlichen Grundlagen und den Aufgaben, die von KMK gemacht worden sind sowie den European Standards and Guidelines (ESG) der European Association for Quality Assurance in Higher Education (ENQUA); vgl. dazu oben C.) III.) 1.).

84 Vgl. insoweit die Vorschläge des Wissenschaftsrates, Empfehlungen (Fn. 12), S. $74 \mathrm{ff}$. 
Zentrale und aufwändige Aufgabe ist allerdings die Akkreditierung und Re-Akkreditierung von Agenturen. ${ }^{85}$ Auch dieses Verfahren folgt dem im Wissenschaftssystem verbreiteten Format von Evaluationsverfahren: ${ }^{86}$ (1) Selbstdarstellung und ggf. Erfahrungsbericht, (2) Begehung und Begutachtung durch eine Gutachtergruppe, die aus einem Mitglied des Akkreditierungsrates ${ }^{87}$ sowie aus vier Vertretern der Wissenschaft, der Studierenden sowie der Berufspraxis besteht, zwei der fünf Mitglieder sollen aus dem Ausland kommen, (3) Stellungnahme der Agentur zur Gutachten- und Beschlussempfehlung, sowie (4) Entscheidung über die Zulassung durch den Akkreditierungsrat, die - im Einklang mit den allgemeinen Strukturen der Akkreditierung - als Verwaltungsakt angesehen wird. ${ }^{88}$ Die (Re-)Akkreditierung von Agenturen ist auf fünf Jahre befristet. Die mit dem Verfahren zusammenhängenden Dokumente werden vom Akkreditierungsrat nach Abschluss des Verfahrens öffentlich bereitgestellt. 89

\section{c. Probleme demokratischer Legitimation}

Verbreitet werden gegen die Stiftung nicht zuletzt aufgrund der Zusammensetzung der Organe erhebliche Bedenken aus Gründen der demokratischen Legitimation der Verwaltung erhoben. ${ }^{90}$ Insbesondere die Benennungsrechte des $\$ 7$ Abs. 2 ASG sind dabei Gegenstand erheblicher Bedenken und dies aus zwei Gründen. Zum einen soll die Zusammensetzung des Akkreditierungsrates unter demokratischen Gesichts-

85 \2 Abs. 1 Nr. 1 ASG; Regeln des Akkreditierungsrates für die Akkreditierung von Agenturen, Drs. AR 91/2009, abrufbar unter http://akkreditierungsrat.de/fileadmin/Seiteninhalte/Startseite/Beschluss_Akkredi-tierung_Agenturen_08_12_09.pdf. Die Akkreditierung erfolgt im Wesentlichen anhand von 7 Kriterien: die Etablierung von verbindlichen internen Standards und Verfahren, die eine konsistente Anwendung der Regeln des Akkreditierungsrates gewährleisten, hinreichende Beteiligung von Wissenschaft, Studierenden und Berufspraxis, eigene Rechtspersönlichkeit, keine Gewinnorientierung, Weisungsfreiheit ihrer Organe, Etablierung eines internen Qualitätsmanagementverfahrens und eines internen Beschwerdeverfahrens sowie eine hinreichende Transparenz und Rechenschaftslegung. Die vom Akkreditierungsrat aufgestellten Kriterien entsprechen den Vorgaben der Standards and Guidelines for Quality Assurance in the European Higher Education Area (ESG). Insoweit ist auch hier eine Europäisierung der Standards zu beobachten.

86 Siehe zum Folgenden Regeln des Akkreditierungsrates für die Akkreditierung von Agenturen, Drs. AR 91/2009, abrufbar unter http://akkreditierungsrat.de/fileadmin/Seiteninhalte/Startseite/Beschluss_Akkredi-tierung_Agenturen_08_12_09.pdf; allgemein auch R. Stockmeyer/W.Meyer, Evaluation, 2010 .

87 Ungeachtet der Tatsache, dass sich dies Zusammensetzung noch in den Regeln findet, wird die Mitgliedschaft eines AR-Mitgliedes offenbar seit 2012 im Interesse einer klaren Trennung von peer review und Entscheidung nicht mehr praktiziert; vgl. Report on the External Evaluation of the German Accreditation Council (Akkreditierungsrat) v. 31. Juli 2013 S. 17.

88 H. Jochum, Verwaltungsverfahrensrecht und Verwaltungsprozeßrecht, S. 469; Th. Mann/D.Immer, Akkreditierung (Fn. 10), S. 347 ; K. Wilhelm, Verfassungs- und verwaltungsrechtliche Fragen (Fn. 10), S. 362.; K. Bieback, Zertifizierung (Fn. 8), S. 186 ff.; D. Immer, Rechtsprobleme (Fn. 10), S. 149 f.

89 Abrufbar unter http://akkreditierungsrat.de/index.php?id=6\&L=1htt...r\%2Fcon\#zulassung.

90 Vgl. dazu D. Immer, Rechtsgrundlagen (Fn. 10), S. 277 ff.; K. Wilhelm, Verfassungs- und verwaltungsrechtliche Grundlagen (Fn.. 10), S. $115 \mathrm{ff}$. 
punkten zu beanstanden sein, zum anderen ruft die Kombination aus Benennung und Bestellung Bedenken hervor. ${ }^{91}$

Der Sache nach wird man den Akkreditierungsrat insoweit den pluralisierten Verwaltungseinheiten zurechnen müssen. ${ }^{92}$ Dabei lässt sich der Akkreditierungsrat nicht ohne weiteres hinsichtlich der Zusammensetzung einem bestimmten Typus pluralisierter Verwaltungseinheiten zurechnen. ${ }^{93}$ Denn seine Zusammensetzung folgt, sieht man von den staatlichen Vertretern ab, dem Gesichtspunkt der Partizipation, in der Aufnahme von Vertretern der Hochschulen und der Studierenden korporativen Aspekten, in dem Vertreter der beruflichen Praxis integriert werden, wie auch der Inkorporation professioneller Expertise, in dem ausländische Vertreter mit Akkreditierungserfahrung einbezogen werden. Diese Auswahl lässt sich als solche durchaus mit der Aufgabe des Akkreditierungsrates rechtfertigen. Dies gilt im Ausgangspunkt auch für die Vertreter der Berufspraxis. Zweifellos repräsentieren diese einen wichtigen stakeholder der Ausbildung. Folgerichtig sind sie auch in die eigentliche Akkreditierung von Studiengängen eingebunden, nicht nur in die Arbeit des Akkreditierungsrates. Die nötige Basislegitimation wird hier zunächst durch das Gesetz vermittelt, dass auch den Modus der Auswahl und Bestellung regeln muss. Ein staatliches Auswahlrecht ist dabei weder stets notwendig noch sinnvoll, da es gerade der Sinn der Pluralisierung sein kann, bestimmte Interessen sich eigenständig artikulieren zu lassen. Korporative und partizipative Beteiligungen sind dafür ein Beispiel. ${ }^{94} \mathrm{Ge}-$ rade für staatsdistanziertere Einrichtungen ist es insoweit nicht zu beanstanden, wenn durch Gesetz die Auswahl der Mitglieder bestimmten Einrichtungen übertragen wird. Das gilt hier jedenfalls für die Auswahl der Vertreter der Hochschulen und Studierenden, die durch ein höherstufiges Repräsentationsorgan der Hochschulen ausgewählt werden. Es ist folglich nicht ein eigenständiger Legitimationszug, der von den Hochschulen über die Hochschulrektorenkonferenz verläuft, der hier die Legitimation etwa im Sinne einer funktionalen Selbstverwaltungseinheit vermittelt, sondern der gesetzlich vorgesehene Auswahlmechanismus, der an die Repräsentationsfunktion einer höherstufigen Einrichtung des Wissenschaftssystems anknüpft und insofern sinnvoll ist. Dies mag auch für die Auswahl von ausländischer Expertise gelten, die gemeinsam der HRK und KMK überlassen wird. Weniger einleuchtend und zudem unterbestimmt ist allerdings die Bestimmung der Vertreter der Berufs-

91 Keine Bedenken ergeben sich ersichtlich hinsichtlich des Stiftungsrates, da auch nach der klassischen Legitimationskonzeption (vgl. zu ihren Grundannahmen H.-H. Trute, Legitimation (Fn. 7) Rn. 4 ff., zu Benennungs- und Entsendungsrechten Rn. 9) jedenfalls eine Mehrheit von durch ununterbrochene Kette von Ernennungsakten legitimierte Mitglieder besteht.

$92 \mathrm{Zu}$ den Anforderungen an die demokratische Legitimation bei diesen Einrichtungen vgl. H.-H. Trute, Legitimation (Fn. 7) Rn. $69 \mathrm{ff}$.

$93 \mathrm{Zu}$ der Typisierung vgl. Th. Groß, Das Kollegialprinzip in der Verwaltungsorganisation, Tübingen 1999, S. $61 \mathrm{ff}$.

94 Vgl. dazu H.-H. Trute, Legitimation (Fn. 7) Rn. 73. 
praxis. ${ }^{95}$ Es ist weder unmittelbar einleuchtend, hier auf die Auswahl von HRK und KMK zu vertrauen, da nicht recht ersichtlich ist, wo die Auswahlexpertise liegen soll. Auch wird man die Ausgestaltung des Status der Mitglieder, etwa im Hinblick auf Unabhängigkeit von Interessenbindungen, Weisungsfreiheit, ${ }^{96}$ Objektivität der Aufgabenerfüllung, für unzulänglich halten können. Insoweit ist die Ausgestaltung durchaus nicht von Bedenken frei.

Als Kooperationsgremium zwischen Hochschulen und Staat unterliegt es keinen Bedenken, dass die eigentliche Benennung einvernehmlich durch die KMK und die HRK erfolgen muss. Kooperationen sind per se dadurch gekennzeichnet, dass beide Seiten zusammenwirken müssen. Insoweit ist auch unter demokratischen Gesichtspunkten nichts gegen einen Einigungszwang auszusetzen, zumal die eigentliche personelle Entscheidung in der im Grundsatz jedenfalls nicht zu beanstandenden Auswahl erfolgt. Die doppelte Rechtsaufsicht durch den Stiftungsrat ebenso wie das zuständige Ministerium in NRW sichert zudem die Rechtmäßigkeit künftiger Entscheidung en hinreichend $\mathrm{ab}$.

Ein eigenes Problem stellen allerdings die vom Akkreditierungsrat erlassenen Regelungen dar. Dabei ist weniger bedeutsam, dass sie ihre Wirksamkeit erst durch die Verträge mit den Agenturen erhalten. Sie wirken - etwa die Regelungen des Verfahren der Akkreditierung - durchaus auf die Hochschulen ein, die sich der zumindest mittelbaren Verpflichtung aus diesen Regelungen vermittelt durch Verträge mit den Agenturen nicht entziehen können. Dies gilt in geringerem Umfang auch für die materiellen Standards, die sich in vielen Hochschulgesetzen allerdings schon als Verpflichtungen der Hochschulen finden und insoweit durch die Standards der Akkreditierung lediglich konkretisiert werden. Gleichwohl ist nicht zu übersehen, dass damit eine Rechtssetzung in einer Grauzone stattfindet, die zwar ebenfalls für den Hochschulbereich nicht untypisch, aber deswegen noch nicht unbedenklich ist. Hier wirkt auch die Tatsache, dass diese Struktur offen und flexibel ausgestaltet werden muss und dies auch im Hinblick auf die notwendige Weiterentwicklung durchaus auch wissenschaftsadäquat ist, nicht mehr überzeugend als Rechtfertigung. Es ist ohne Weiteres möglich, die Verfahrensgestaltung durch den Akkreditierungsrat gesetzlich weitergehend vorzuzeichnen. Auch für die Strukturvorgaben gilt, dass sie zumindest in den Ländern, in denen die Kriterien sich nicht schon in den Hochschulgesetzen finden, durchaus eine Wirkung bekommen, die den früheren Rahmenprüfungsordnungen als Empfehlungen rechtlich nicht zukam. Die notwendige Koordination im Bildungsföderalismus muss nicht als Legitimation für rechtliche Grau-

95 Insoweit auch D. Immer, Rechtsgrundlagen (Fn. 10), S. 323.

96 Allerdings enthält $\ 6$ Abs. 3 der Satzung der Stiftung eine Regelung der Weisungsfreiheit der Mitglieder des Akkreditierungsrates unbeschadet der Rechtsaufsicht des Stiftungsrates. Ungeachtet der Frage, ob dies nicht einer gesetzlichen Ausgestaltung des Status bedürfte, dürfte dies allein Weisungen des Stiftungsrates betreffen. 
zonen taugen. Auch hier gilt, dass die in diesem Bereich ungeliebte staatsvertragliche Konstruktion einen deutlichen demokratischen und rechtsstaatlichen Mehrwert hat.

\section{Agenturen}

Die Akkreditierung von Studiengängen erfolgt durch zehn Akkreditierungsagenturen, die entweder einen fachbezogenen oder regionalen Schwerpunkt haben, die allerdings weder gebietliche noch fachliche Monopole haben. Sie stehen vielmehr im Wettbewerb untereinander, ${ }^{97}$ der Preis- und Qualitätswettbewerb sein soll. Die Agenturen, von denen immerhin drei aus dem Ausland stammen, haben unterschiedliche Rechtsformen gewählt. ${ }^{98}$ Alle Agenturen haben Hochschulen, Fachgesellschaften und -verbände sowie Arbeitgeberverbände und Gewerkschaften in ihre Organisations- und Entscheidungsstrukturen, in ihre Gremien eingebunden. ${ }^{99}$ Für alle Agenturen gilt zudem, dass sie nicht gewinnorientiert, aber auf Vollkostenbasis arbeiten dürfen. ${ }^{100}$

\section{a. Agenturen als Beliehene?}

Für die Frage, ob das System nun staatsnah und öffentlich-rechtlich oder staatsdistanzierter und zivilrechtlich jedenfalls im Hinblick auf die Stellung der Agenturen ausgerichtet ist, spielt in der Diskussion eine erhebliche Rolle, ob die Agenturen Beliehene sind. Grundsätzlich kann diese Frage auf der Ebene des Verhältnisses von Akkreditierungsrat und Agenturen, aber natürlich auch im Verhältnis von Landeshochschuladministration und Agenturen nach Maßgabe der jeweiligen Landeshochschulgesetze gestellt werden.

Das ASG enthält textlich keinen Hinweis auf eine Beleihung. Insbesondere die Norm des $\mathbb{2}$ Abs. 1 Nr. 1 ASG, die die (Re-)Akkreditierung von Akkreditierungsagenturen durch eine befristete Verleihung der Berechtigung, Studiengänge und hochschulinterne Qualitätssicherungssysteme durch Verleihung des Siegels der Stiftung zu akkreditieren, gibt in der Sache dafür nichts her. Zum einen handelt es sich bei der als Stiftungszweck überschriebenen Norm um eine Aufgaben- und nicht um eine Befugnisnorm. Das Gesetz aber erteilt weder hier noch an anderer Stelle eine Ermächtigung

97 Hierin sieht der Wissenschaftsrat eine deutliche Differenz zu anderen ausländischen Qualitätssicherungssystemen; vgl. Wissenschaftsrat, Empfehlungen (Fn. 12), S. 95 f.

98 Die Agenturen ACQUIN, AHPGS, AQUAS und ASIIN sind Vereine gemäß $\$ 21 \mathrm{ff}$. BGB, AKAST ist ein Verein nach kirchlichem Recht, AQA ist ein Verein nach dem Österreichischen Vereinsgesetz. Die Evaluationsagentur Baden-Württemberg (evalag) ist eine Stiftung des öffentlichen Rechts, die ursprünglich als Zusammenschluss der niedersächsischen Hochschulen gegründete - Zentrale Evaluations- und Akkreditierungsagentur Hannover (ZEvA) ist eine Stiftung bürgerlichen Rechts, die Agentur FIBAA ein Stiftung nach Schweizer Recht.

99 Vgl. etwa die vier Mitgliedergruppen der Akkreditierungsagentur für Studiengänge der Informatik, der Naturwissenschaften und der Mathematik e. V. (ASIIN): Technische und naturwissenschaftliche Vereine sowie berufsständische Organisationen, Wirtschaftsverbände und Spitzenverbände der Sozialpartner, Universitäten, Fachhochschulen (http://www.asiin-ev.de/pages/de/asiin-e.-v/ueber-uns/ mitglieder.php).

100 Vgl. Akkreditierungsrat, Regeln für die Akkreditierung von Agenturen, Beschluss des Akkreditierungsrates v. 8.12.2009 i.d.F. v, 10.12.2010, Drs. AR 86/2010 Nr. 2.3.2. 
zu einer Beleihung Privater. Man müsste andernfalls annehmen, dass in dem Akkreditierungsakt selbst eine Beleihung steckt. Auch wenn dieser unstrittig einen Verwaltungsakt darstellt, sagt dies nichts über das Verhältnis zwischen den Agenturen und den zu akkreditierenden Einrichtungen aus. ${ }^{101}$ Allein die Begriffswahl der Akkreditierung, das zeigen schon die oben vorgestellten Bereiche der Qualitätssicherung, sagt darüber nichts aus. Insoweit kann auch aus dem Verhältnis von Akkreditierungsrat und Agenturen letztlich wenig für diese Frage gewonnen werden, ebenso wenig wie aus der Tatsache, dass wesentliche Normen, die nicht nur das Akkreditierungssystem regeln, sondern auch die Maßstäbe der Akkreditierung vorgeben, öffentlich-rechtlicher Natur sind. Denn das ist für das Akkreditierungsmodell nichts Ungewöhnliches. ${ }^{102}$ Auch kann es nicht darauf ankommen, in welcher Sphäre die Agenturen handeln, in der des Staates oder der der Hochschulen. ${ }^{103}$ Dies setzt voraus, was man letztlich beantworten will. Ebenso wenig ergiebig ist die Tatsache, dass damit ein einseitiges Handeln und damit einhergehend auch einseitige Begründungen von Wirkungen erfolgen. ${ }^{104}$ Das ist in anderen Bereichen, die auch vom Gesetzgeber als eindeutig privatrechtlich verstanden werden, durchaus der Fall - wie nicht zuletzt der Bereich der Produktakkreditierungen ebenso zeigt ${ }^{105}$ wie die oben referierte sozialrechtliche Problematik. Das macht private Akkreditierer nicht zu öffentlichrechtlich Handelnden. Mehr noch gilt dies für den Versuch, aus den Wirkungen gegenüber den Hochschulen einen Verwaltungsakt zu konstruieren, die Frage nach der Behördeneigenschaft letztlich aber darüber zu beantworten, dass auch eine nicht rechtswirksam vorgenommene Beleihung einen Rechtsschein erzeugt. ${ }^{106}$ Eine Auslegung der Vorschriften des ASG soll dies ergeben, ergibt es aber der Sache nach gerade nicht, einmal abgesehen davon, dass dies ohnehin keine „bundesweite“ Beleihung sein könnte. ${ }^{107}$ Der Sache nach erfolgt die Beleihung ohnehin rechtssubjektbezogen, nicht handlungsbezogen. Sie vermittelt allererst die Zuordnung zur Verwaltung und damit eine etwaige Behördeneigenschaft. Es dürfte sich um einen Zirkelschluss handeln, von einer etwaigen Verbindlichkeit der Akkreditierung gegen-

101 Vgl. dazu oben B.

102 Vgl. aber D. Immer, Rechtsprobleme (Fn. 10), S. 232 ff., der freilich selbst zum Zwischenergebnis kommt, dass die Frage offen ist.

103 Dazu etwa D. Immer, Rechtsprobleme (Fn. 10), S. 228 f.

104 Vgl. D. Immer, Rechtsprobleme (Fn. 10), S. $229 \mathrm{ff}$.

105 Vgl nur M. Eifert, Die geteilte Kontrolle - Die Beteiligung Privater an der Rechtsverwirklichung, DV 39 (2006), S. 309 (311).

106 So jüngst wieder die Lösung von D. Immer, Rechtprobleme (Fn. 10), S. 217 ff., 255.

107 Vgl. aber D. Immer, Rechtsprobleme (Fn. 10), S. 254 f., der für die bundesweite Qualifizierung auf das Verwaltungsabkommen abstellen möchte ("flankiert“). Einmal abgesehen davon, dass dies kaum die je ländereigenen Anforderungen an den institutionellen Gesetzesvorbehalt überspielen könnten, was den Ländern gewiss nicht unbekannt sein dürfte, stellt es auch die Frage, warum dann Länder, die wie Nordrhein-Westfalen eine Beteiligungslösung präferieren, dieses in ihren Landeshochschulgesetzen regeln wollen. Umgekehrt gilt auch: Warum sollte eine Beleihung erfolgen, wenn Länder sich noch eine abschließende Letztentscheidung vorbehalten. Eine wenig plausible, Widersprüche erst generierende Lösung. 
über den Hochschulen auf eine Beleihung durch den Akkreditierungsrat zu schließen. ${ }^{108}$

Hochschulrektorenkonferenz, ${ }^{109}$ Akkreditierungsrat ${ }^{110}$ und Agenturen ${ }^{111}$ gehen gleichermaßen von einem staatsfernen System der Akkreditierung aus, nicht anders als die Begründung zum ASG. ${ }^{112}$ Schon im Hinblick auf die Frage, ob die Stiftung auch Akkreditierung von Studiengängen übernehmen soll, wird darauf verwiesen, dass die Mitwirkung der Länder in der Stiftung dem Prinzip des peer review widerspreche, das der Akkreditierung der Studiengänge zugrunde liege. ${ }^{113}$ Dies entspricht der Struktur des Gesetzes, nicht zuletzt der vom Gesetz selbst betonten Staatsferne und derjenigen der gewollten Internationalisierung. So finden sich schon in dem noch zu behandelnden Mustervertrag des Akkreditierungsrates Verpflichtungen der Agenturen, die darauf gerichtet sind, in den Verträgen zwischen den Agenturen und den Hochschulen sicherzustellen, dass bestimmte Systemvorgaben bis hin zur Entziehung der Akkreditierung umgesetzt werden können. ${ }^{114}$ Dessen bedürfte es nicht, wenn ohnehin schon aufgrund der Behördeneigenschaft der Agenturen von den Befugnissen des VwVfG NRW Gebrauch gemacht werden könnte. Darüber hinaus ist das Gesetz auf den Wettbewerb der Agenturen ausgerichtet und darauf, dass diese sich

108 So aber ersichtlich das Vorgehen von D. Immer, Rechtsprobleme (Fn. 10), S. 225 ff., wobei undeutlich bleibt, auf welche öffentlich-rechtlichen Normen im Verhältnis von Akkreditierungsrat und Agenturen er abstellen möchte, um eine Beleihung zu begründen. Eher erscheint es so, als würde aus der Entscheidung der Agenturen zur Siegelverleihung wegen der vorgeblich öffentlich-rechtlichen Handlung dann auf einen Verwaltungsakt und damit eine rechtliche oder faktische Beleihung geschlossen.

109 Hochschulrektorenkonferenz, Beschluss v. 14.10.2008. Durch den Beschluss der HRK zur Weiterentwicklung des Akkreditierungssystems vom 24.4.2012, der für ein Qualitätsaudit plädiert, wird die Staatsferne noch einmal deutlicher akzentuiert und der Akkreditierungsrat zu einem Qualitätsrat und die Agenturen zu Beratungseinrichtungen der Hochschulen gemacht. Insoweit fehlte es dann an jeglichem Hinweis zur Ausgestaltung eines hoheitlichen Systems - jetzt auch für den Akkreditierungsrat. Eine ganz andere Frage ist, ob von Seiten der Politik die Bereitschaft besteht, in naher Zukunft allein auf die Selbstorganisation und Verantwortung der Hochschulen abzustellen. Die derzeitigen Gesetzesvorhaben weisen eher in die gegenteilige Richtung.

110 Akkreditierungsrat, Stellungnahme zur Weiterentwicklung des Akkreditierungssystems, Beschluss v. 14.11.2011, Drs AR 1/2011, S. 3 f.

111 Vgl. etwa ACQUIN, http://www.asiin-ev.de/pages/de/asiin-e.-v/programmakkreditierung/allgemeine-kriterien-und-feh.php mit der Betonung von Staatsferne und Unabhängigkeit; ASIIN e.V., Allgemeine Kriterien für die Akkreditierung von Studiengängen, S. 46, http://www.asiin-ev.de/pages/de/ asiin-e.-v/programmakkreditierung/allgemeine-kriterien-und-feh.php; sowie AQUAS, Stellungnahme des Vorstands zum Referentenentwurf des Hochschulzukunftsgesetzes NRW v. 4.02.2014; weitere Aspekte finde sich bei D. Immer, Rechtsprobleme (Fn. 3), S. $218 \mathrm{f}$.

112 LT-Drucks. 13/6182 S. 12. Missverständlich wiedergegeben bei D. Immer, Rechtsprobleme (Fn. 10), S. 219. Die entsprechende Passage der Begründungen nimmt ihren Ausgangspunkt von den privatrechtlich handelnden und organisierten Agenturen und macht dann erst die nicht inhibierte Vergabe anderer Siegel zum Thema und verlangt sodann für diesen Bereich die Lauterbarkeit und nicht etwa umgekehrt, wie es bei D. Immer scheint. Das entspricht offenbar auch dem Verständnis durch den Akkreditierungsrat.

113 NW LT-Drucks 13/6182 S. 10 zu $\$ 2$ ASG.

114 Vgl. etwa $\$ 7$ Abs. 3 der Mustervereinbarung zwischen dem Akkreditierungsrat und den Agenturen, Drs. AR 97/2009. 
über Entgelte finanzieren, die ebenfalls der autonomen Vereinbarung unterliegen. Dies dürfte sich schwerlich mit einer Beleihung vereinbaren lassen. ${ }^{115}$

Die „European Standards and Guidelines for Quality Assurance in the European Higher Education Area“116 postulieren zudem die Unabhängigkeit der Akkreditierungsagenturen von Dritten. ${ }^{117}$ Das umfasst die operationelle Unabhängigkeit von Hochschulen wie Ministerien. Die Beachtung dieser Anforderungen ist wiederum Voraussetzung für die Aufnahme in das EQAR Register, ${ }^{118}$ das wiederum die Beachtung der ESG sichert und damit Basis für eine wechselseitige Anerkennung von Qualitätssicherungen sein kann. Eine solche operationelle Unabhängigkeit von dem Akkreditierungsrat ist in den Fällen einer Beleihung indes nicht gewährleistet. ${ }^{119}$ Von daher gibt es gute Gründe dafür anzunehmen, dass jedenfalls durch das ASG eine Beleihung nicht erfolgt.

Indes ist nicht zu übersehen, dass vereinzelt Landesgesetze eine Beleihung der Agenturen für den Fall der Tätigkeit für die landeseigenen Hochschulen vorsehen oder zumindest vorsehen wollen. ${ }^{120}$ Darüber hinaus soll aus den Landesgesetzen die öffentlich-rechtliche Lösung abgeleitet werden, die sich letztlich aus der Akkreditierungspflicht und der Verbindlichkeit der Entscheidung von Agenturen für die Hochschulen ${ }^{121}$ oder die staatlichen Genehmigungsbehörden ergeben. ${ }^{122}$ Indes zeigt sich, und darauf wird zurückzukommen sein, dass es letztlich um Formen wissenschaftsadäquater Qualitätssicherung geht. Von einer Beleihung wird man daher nicht ausgehen können.

115 Dies sieht auch D. Immer, Rechtsprobleme (Fn. 3), S. 219 ff. so, möchte dies indes durch den Hinweis auf die Gemeinnützigkeit entschärfen. Indes ist die Gemeinnützigkeit mit der Frage des Rechtsregime zunächst einmal nicht verknüpft und der Hinweis auf eine deshalb möglicherweise fehlende Grundrechtsträgerschaft der Agenturen ist, wie gezeigt, durchaus zweifelhaft, weil von einem überholten Verständnis von Gemeinnützigkeit ausgehend; vgl. dazu oben unter C.) I.).

116 Vgl. dazu oben unter C.).

117 European Standards and Guidelines for Quality Assurance in the European Higher Education Area, 3.6.

118 EQAR, Procedures for Application, 2012, 2.2., in dem alle Akkreditierungseinrichtungen des Europäischen Hochschulraumes eingetragen werden können, eine Grundlage für die spätere wechselseitige Anerkennung von Akkreditierungen.

119 Vgl. dazu die skeptischen Anmerkungen zur Frage, ob die Ausgestaltung des Akkreditierungsrates in Fragen der Akkreditierung von Agenturen den gestellten Anforderungen genügen, Report on the External Evaluation of the German Akkreditation Council (Akkreditierungsrat) v. 31. Juli 2013, S. 30 ff. Weniger skeptisch Wissenschaftsrat, Empfehlungen (Fn. 12), S. 63.

120 In keinem Hochschulgesetz findet sich freilich derzeit eine explizite Beleihungslösung. Allerdings wird für den bisherigen $\$ 7$ Abs. 2 HG NW eine solche aus dem Gesetz entnommen; vgl. OVG NW Beschluss v. 13.11.2009 - 15 E 1153/09 - juris- Rn. 10. Der Entwurf eines neuen Hochschulgesetzes sieht in $\mathbb{}$ 7a E-HG NW nunmehr in der Tat eine Beleihungslösung vor, die insoweit auch einen Kommentar zu der bisherigen Rechtslage nach dem ASG und dem HG NW abgibt.

121 Vgl. dazu oben C.)III.) 2.) a.).

122 Vgl. zur Verbindlichkeit als entscheidendem Kriterium D.Immer, Rechtsprobleme (Fn. 10), S. 229 ff.; eine Verbindlichkeit sowohl für die Hochschulen wie für die staatlichen Behörden im Kontext der Klageart diskutierend K. Wilhelm, Verfassungs- und verwaltungsrechtliche Fragen (Fn. 10), S. $341 \mathrm{ff}$. 


\section{b. Agenturen als Grundrechtsberechtigte oder -verpflichtete?}

Unklar ist demgemäß auch, ob und inwieweit sich die Agenturen auf Grundrechte berufen können. Dies wird aus verschiedenen Gründen bestritten. Ganz wesentlich wird jedenfalls hinsichtlich des Art. 12 Abs. 1 GG ins Feld geführt, dass die Agenturen als gemeinnützige Vereine nicht der Berufsfreiheit unterfallen können, so dass diesen letztlich, wenn überhaupt, die Berufung auf Art. 2 Abs. 1 GG bliebe. ${ }^{123}$

Indes wird man kaum übersehen können, dass die Agenturen diese Tätigkeit als eine auf Dauer ausgerichtete ausüben, die auch der Erhaltung einer Lebensgrundlage, nicht zuletzt für die bei ihr angestellten Personen, dient. ${ }^{124}$ Auch ist die fehlende Gewinnorientierung oder die bestehende Gemeinnützigkeit nicht mit ehrenamtlicher, unbezahlter Arbeit zu identifizieren. Vielmehr gibt es eine Vielzahl von gemeinnützigen Einrichtungen des Wirtschafts-, Sozial- aber auch des Hochschulbereichs, die ebenso einer Vielzahl von Menschen als Grundlage der Berufstätigkeit dienen und auch wirtschaftlich kostendeckend arbeiten müssen. So liegt es auch hier; die fehlende Gewinnorientierung der Tätigkeit im Rahmen des ASG ist durchaus mit einer Vollkostenorientierung verbunden.

Folgerichtig wird die Frage der Gemeinnützigkeit als Ausschlussgrund für die Berufung auf Art. 12 Abs. 1 GG auch in einer Reihe von Urteilen der jüngeren Zeit nicht mehr eigens thematisiert. Vielmehr wird von der Anwendbarkeit des Art. 12 Abs. 1 GG ausgegangen. So hat bereits die 3. Kammer des 1. Senats des Bundesverfassungsgerichts in ihrem Beschluss vom 7. November 2001 ausgeführt, dass die in Art. 12 Abs. 1 GG gewährleistete Berufsfreiheit jede auf Dauer angelegte und auf Erwerb gerichtete Tätigkeit umfasse, der grundrechtliche Schutz gemäß Art.19 Abs. 3 GG auch für Vereine gelte, sofern die Führung eines Geschäftsbetriebs zu ihren satzungsgemäßen Zwecken gehöre, und für die Anwendung des Art. 12 Abs. 1 GG auf ihre erwerbsmäßige Tätigkeit, die eine Gewinnerzielung nicht voraussetze, nicht maßgeblich sei, ob die Vereine dabei gemeinnützig wirkten. ${ }^{125}$ Auch das Bundesverwaltungsgericht ist von der Anwendbarkeit des Art. 12 Abs. 1 GG auf einen als gemeinnützig anerkannten Verein mit der Begründung ausgegangen, dass ein solcher Verein zwar nicht mit Gewinnerzielungsabsicht betrieben werden dürfe, dies jedoch nichts daran ändere, dass dieser geschäftsmäßig betrieben werde und kostendeckend arbeiten solle, sodass bei ihm der für Art. 12 Abs. 1 GG erforderliche ökonomische

123 Vgl. etwa J. Lege, Akkreditierung Studiengänge (Fn. 10), S. 707; Th. Mann, VVDStRL 65 (2006), S. 331 (332); D. Immer, Rechtsprobleme (Fn. 10), S. 260 ff. Allgemein zur Frage der Gemeinnützigkeit im Kontext von Art. 12 GG vgl. J. Wieland, in: H. Dreier (Hrsg.), GG Art. 12 Rn. 42; R. Breuer, Freiheit des Berufs, in: J. Isensee/P. Kirchhof (Hrsg.), HStR VIII, Heidelberg 2010, $\mathbb{1 7 0 ~ R n . ~ 6 9 ; ~}$ H. D. Jarass, in: H. D. Jarass/B. Pieroth (Hrsg.), GG, München 2011, Art. 12 Rn. 6; W. Berg, GewArch 1977, $269 \mathrm{ff}$.

124 Zum Berufsbegriff in diesem Sinne vgl. BVerfGE 7, 377 (397); 54, 301 (313) std. Rspr. BVerfGE 103, 172 (182 Rn. 29); BVerwGE 94, 269 (277).

125 BVerfG NJW 2002, 2091; offen gelassen vom 2. Senat des BVerfG DVBl. 2007, 1555 (1559). 
Grundbezug nicht fehle. ${ }^{126}$ Entscheidend kommt es also nicht auf das Merkmal der Gemeinnützigkeit an, sondern darauf, dass eine Tätigkeit geschäftsmäßig und kostendeckend betrieben werden muss und dass damit eine ökonomische Einbettung der gesamten Tätigkeit erfolgt. Dafür spricht im Übrigen auch, dass das ASG einen Wettbewerb der Agenturen auslösen wollte und damit - auch im Rahmen der fehlenden Gewinnorientierung - einen ökonomisch motivierten Funktionsmechanismus sich zunutze machen wollte, um zumindest einen Qualitäts-, aber auch einen Preiswettbewerb für die Zwecke des Gesetzes zu nutzen. ${ }^{127}$

Auch wenn man annehmen wollte, dass Akkreditierungsagenturen nach Maßgabe des Verständnisses einzelner landesrechtlicher Regelungen als Beliehene arbeiten, würde dies nicht vollumfänglich gegen den Schutz des Art. 12 Abs. 1 GG sprechen. Dies gilt jedenfalls hinsichtlich der Akkreditierung als solche. Insoweit unterfällt die Ausgestaltung der Akkreditierungsvorschriften dem Schutzbereich des Grundrechts. Hier gilt nichts anderes als für die staatlich gebundenen Berufe. Dies gilt umso mehr, als die Ausübung der Akkreditierungstätigkeit jenseits der vom ASG geregelten Tätigkeit, etwa im internationalen Bereich, letztlich von dem Zugang zu der Akkreditierung durch den Akkreditierungsrat abhängt. ${ }^{128}$ Anderes würde auf der Grundlage der Annahme einer Beleihung für die Ausübung der Akkreditierungstätigkeit durch die Agenturen gelten. Insoweit wären sie unter der Voraussetzung der Annahme einer Beleihung grundrechtsverpflichtet.

Neben dem Erfordernis einer zureichenden Rechtsgrundlage für die Handlungen des Akkreditierungsrates, hat dies zur Folge, dass die Inanspruchnahme einseitiger Gestaltungsbefugnisse im Rahmen der Ermessensausübung die grundrechtliche Stellung der Beteiligten berücksichtigen muss.

\section{Zum Verhältnis von Akkreditierungsrat und Agenturen}

Dies wirkt auf das Verhältnis von Akkreditierungsrat und Agenturen ein. Dem Akkreditierungsrat kommt - wie dargelegt - eine Scharnierfunktion an der Schnittstelle von Strukturverantwortung der Länder und Prozessverantwortung der Agenturen für die Durchführung der Akkreditierungsverfahren zu. ${ }^{129}$ Der Sache nach wird das Verhältnis zwischen dem Akkreditierungsrat und den Agenturen durch die zentrale Kooperationsverpflichtung nach Maßgabe des $\$ 3$ Abs. 1 ASG gebildet. Diese soll eine die gegenseitigen Rechte und Pflichten abbildende Grundlage in dem zwischen

126 BVerwGE 95, 15, 20; vgl. auch VGH BW, Beschluss v. 4.3.2014 - 10 S 1127/13 - juris Rn. 20; LSG MV, Beschluss v. 30.8.2012 - L 9 SO 1/10 - juris Rn. 51; BVerwG, Urteil v. 30.8.2012 - 3 C 17/11 - juris Rn. 1, 43 ff. = BVerwGE 144, 109 ff.; OVG Lüneburg, Beschluss v. 9.7.2010 - 4 ME 306/09 - juris Rn. 6.

127 Die Vollkostenorientierung schließt einen Wettbewerb nicht aus, sondern erlaubt ebenfalls einen Preiswettbewerb auf der Basis von Effizienz; anders ersichtlich D. Immer, Rechtsprobleme (Fn. 10), S. 221.

128 In diesem Sinne auch D. Immer, Rechtsprobleme (Fn. 10), S. 263 ff.

129 NW LT-Drucks. 13/6182 S. 9. 
Agenturen und Akkreditierungsrat zu schließenden Vertrag finden, dessen Gegenstände dann in $\$ 3$ Abs. 2 ASG genannt sind. Gesetzlich vorgesehen ist also nicht etwa ein hierarchisches Unterstellungsverhältnis, wie es einer Beleihung entsprechen würde, sondern ein Kooperationsverhältnis zwischen dem Akkreditierungsrat und den Agenturen. ${ }^{130}$ Das Gesetz spricht insoweit von der vertrauensvollen Zusammenarbeit ( $\$ 3$ Abs. 1 ASG). Diese Vereinbarungen sind konsequenterweise als öffentlich-rechtliche Verträge einzuordnen. ${ }^{131} \mathrm{Um}$ ein überwachungsfreies Tätigwerden einer Agentur bei der Vergabe von Siegeln auszuschließen, ist die Wirksamkeit der Akkreditierung vom Abschluss einer Vereinbarung gemäß $\$ 3$ ASG abhängig. ${ }^{132}$

\section{a. Die Regelungen des Mustervertrages}

In der Vereinbarung werden in Konkretisierung der gesetzlichen Vorgaben des $\mathbb{3}$ Abs. 2 ASG natürlich auch und vor allem die Beachtung der der staatlichen Verantwortung entspringenden Strukturvorgaben ebenso wie die Einhaltung von Mindestanforderungen für das Akkreditierungsverfahren, dessen Regelung dem Akkreditierungsrat gemäß $\mathbb{2}$ Abs. 1 Nr. 3 ASG obliegt, sichergestellt. Insoweit werden die Strukturvorgaben in vertragliche Verpflichtungen transformiert. Dies erlaubt die Wahrnehmung der Verantwortung für die Vergleichbarkeit von Abschlüssen im Bundesstaat wie auch eine sachgerechte Aufgabenverteilung zwischen dem Rat und den Agenturen. Insoweit spricht das Gesetz mit Bedacht von Mindestanforderungen, deren Beachtung im Vertrag gesichert werden muss. Dies folgt konsequent dem Modell der Abschichtung von Struktur- und Prozessverantwortung. Schon um der Erhaltung der Lernfähigkeit und Flexibilität im Hinblick auf veränderte Anforderungen willen, ist die Prozessverantwortung der Agenturen (also vor allem die Ausgestaltung des peer review) Teil der Prozessverantwortung, die aus Qualitätssicherungsgründen durch einen Verfahrensrahmen eingegrenzt wird, um auch Vergleichbarkeit der Verfahren in den Grundlagen sichern zu können. Es wird also gerade nicht eine Verfahrensvollregelung durch den Rat vorgesehen, sondern nur eine Regelung von Mindestanforderungen, um die Funktionsfähigkeit des Systems der Akkreditierung sicherzustellen. ${ }^{133}$ Ebenso muss die Lauterbarkeit des Umgangs mit dem Siegel der Stiftung schon deshalb sichergestellt werden, weil das Gesetz selbst eine Tätigkeit der Agenturen auch außerhalb des durch den Akkreditierungsrat beaufsichtigten Bereichs erwartet, die als gewerbliche Tätigkeit der Agenturen in Konflikt mit den Anforderungen des Akkreditierungsrates geraten kann. ${ }^{134}$

130 Siehe $\mathbb{3}$ Abs. 1 ASG: „Die Stiftung arbeitet mit den Agenturen vertrauensvoll zusammen [...]“; \1 Abs. 1 der Mustervereinbarung (http://akkreditierungsrat.de/fileadmin/Seiteninhalte/Beschluesse_AR/Musterver-trag_08_12_09.pdf) spricht von „Partner[n] im Akkreditierungssystem“.

131 Offen gelassen bei K. Wilhelm, Verfassungs- und verwaltungsrechtliche Fragen (Fn. 10), S. 358 ff.

132 Regeln des Akkreditierungsrates für die Akkreditierung von Agenturen, Drs. AR 91/2009, S. 6.

$133 \mathrm{Zu}$ den Strukturierungsdefiziten vgl. oben C.) III.) 1.).

134 NW LT-Drucks. 13/6182 S. 12. 
Darüber hinaus werden etwa Konsultations- und Berichtspflichten des Akkreditierungsrates gegenüber den Agenturen ebenso wie Informations- und Meldepflichten der Agenturen gegenüber dem Akkreditierungsrat geregelt. So eröffnet etwa die Pflicht der Agenturen, dem Akkreditierungsrat negative Akkreditierungsentscheidungen durch die Agenturen zu melden, ${ }^{135}$ diesem die erforderlichen Kontrollmöglichkeiten. Der Akkreditierungsrat sichert sich zudem ein umfassendes Auskunftsrecht gegenüber den Agenturen ebenso wie die Möglichkeit, an allen Verfahren und Gremiensitzungen teilzunehmen. ${ }^{136}$ Zur Überwachung führt er sowohl anlassbezogene, wie auch anlassunabhängige stichprobenartige Prüfungen einzelner Akkreditierungsverfahren der Agenturen durch. ${ }^{137}$ Er kann die Agenturen zur Aufhebung einer Entscheidung gegenüber einer Hochschule verpflichten, wenn diese die Akkreditierungskriterien nicht sachgerecht angewandt hat oder verfahrensfehlerhaft ist. ${ }^{138}$ Zudem steht ihm gegenüber den Agenturen vertraglich ein gestuftes Sanktionssystem zur Verfügung, dass von einer Vertragsstrafe über eine Verkürzung der Akkreditierungsfrist, bis hin zum Entzug der Akkreditierung - also dem Widerruf der Zulassung - reicht. ${ }^{139}$

Im Ergebnis jedenfalls verfolgt das ASG die Linie einer vertrauensvollen, kooperativen Aufgabenerfüllung durch eine klare Abschichtung von Verantwortlichkeiten, Regelungsmöglichen und einer Verbindlichmachung durch eine vertragliche Gestaltung des Verhältnisses zwischen Agenturen und Rat.

\section{b. Der Mustervertrag zwischen Hierarchie und Kooperation}

Wie schwer die Balance zu halten gleichwohl fällt und wie leicht von dem Modell der Kooperation in den Modus hierarchischer Bestimmung umgewechselt werden kann, zeigt allerdings ebenfalls der Mustervertrag. Deutlich wird dies in $\$ 5$ der Vereinbarung. Dieser enthält über die selbstverständliche Regelung, dass die Agenturen sich zur Anwendung der ländergemeinsamen Strukturvorgaben und der landespezifischen ergänzenden oder ersetzenden Strukturvorgaben verpflichten, hinaus in $\mathbb{} 5$ Abs. 2 die Verpflichtung zur Anwendung aller vom Akkreditierungsrat in Umsetzung des $\mathbb{2}$ ASG beschlossenen Vorgaben. Die Verpflichtung wird zudem dynamisiert und die Nichteinhaltung äußerstenfalls mit dem Entzug der Akkreditierung sanktioniert. Dies ist eine durchaus weitreichende Klausel, die das Kooperationsverhältnis, wie es vom Gesetz vorgesehen ist, in ein hierarchisches Verhältnis transformiert. Diese Klausel beseitigt zudem jede Art von Vertrauensschutz durch die Akkreditie-

$135 \$ 9$ Abs. 6 S. 1 der Mustervereinbarung (Fn. 133).

$136 \$ 13$ Abs. 1 der Mustervereinbarung (Fn. 133).

137 Im Einzelnen Verfahren des Akkreditierungsrates zur Überprüfung der seitens der Agenturen durchgeführten Akkreditierungen, Drs. AR 100/2009, abrufbar unter http://akkreditierungsrat.de/filead$\mathrm{min} /$ Seiteninhalte/Be-schluesse_AR/Ueber-pruefung_08_12_09.pdf. Danach werden im Regelfall vier Verfahren pro Jahr und Agentur geprüft.

$138 \$ 7$ Abs. 1 der Mustervereinbarung (Fn. 133).

$139 \$ 15$ Abs. 1 der Mustervereinbarung (Fn. 133). 
rung und macht der Sache nach die Agenturen zu einer Art nachgeordnete Einrichtungen des Akkreditierungsrates, mit der Möglichkeit des Erlasses von im Beschlusswege durchgesetzten Vorschriften des Akkreditierungsrates für die Agenturen. Die

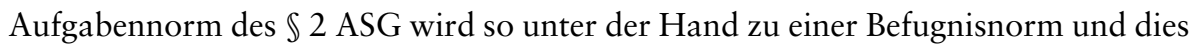
ohne die Abstufungen des Regelungsgehalts des $\mathbb{2}$ ASG zu beachten: die Differenzierung der Bedeutung der Aufgaben beider Absätze, ${ }^{140}$ der unterschiedliche Umfang der Gestaltungsbefugnis zwischen Zusammenfassung der Strukturvorgaben, und Regelung des Akkreditierungsverfahrens ( $\$ 2$ Abs. 1 Nr. 3 ASG), allerdings auf Mindestvoraussetzungen begrenzt, zwischen hinwirken und fördern. Dieser sehr unterschiedliche Regelungsgehalt ist daher schon bei der Auslegung dieser Klausel des Mustervertrages zu berücksichtigen.

Darüber hinaus wird man die Klausel indes, soweit man sie nicht aufgrund der Ausnutzung einer einseitigen Bestimmungsmacht für treuwidrig hält, jedenfalls einer inhaltlichen Kontrolle unterwerfen müssen. Die Vertragslösung soll im Prinzip einen angemessenen Interessenausgleich garantieren. Dies setzt freilich voraus, dass die Bedingungen der Selbstbestimmung auch tatsächlich gegeben sind. ${ }^{141}$ Nur dann lässt der zum Ausdruck gebrachte übereinstimmende Wille der Vertragsparteien auf einen sachgerechten Interessenausgleich schließen. ${ }^{142}$ Anders ist dies dort, wo aufgrund ungleicher Verhandlungspositionen einer der Vertragspartner ein solches Gewicht hat, dass er den Vertragsinhalt faktisch einseitig bestimmen kann. Dann ist es Aufgabe des Rechts auf die Wahrung der Grundrechtspositionen der beteiligten Parteien hinzuwirken, um zu verhindern, dass sich für einen Vertragsteil die Selbstbestimmung in eine Fremdbestimmung verkehrt. ${ }^{143}$

Im vorliegenden Kontext verwendet der Akkreditierungsrat einen Mustervertrag und macht dessen Abschluss ohne weitere Verhandlungsbereitschaft zur Voraussetzung der Akkreditierung und räumt sich in einer vertraglichen Regelung ein, den Regelungsgehalt des Vertrages im Wesentlichen unkoordiniert einseitig ändern zu können. Das dürfte schon mit dem gesetzlich verankerten Kooperationsprinzip kaum vereinbaren sein. Auch hier wirkt dies über den Hebel der Entziehung der Akkreditierung letztlich als eine hierarchische Unterordnung durch unbillige Ausnutzung der Verfügung über den Berufszugang. Insoweit bedürfte es bei der Inanspruchnahme der Klausel des $\ 5$ der Mustervereinbarung einer verstärkten gerichtlichen Kontrolle der in Anspruch genommenen Befugnisse des Akkreditierungsrates.

140 Die Begründung spricht insoweit von einem Kernbestand von Aufgaben, der in $\$ 2$ Abs. 1 ASG geregelt wird; vgl. NW Drucks 13/6182, S. 10.

141 Für zivilrechtliche Verträge vgl. BVerfGE 81, 242 (254f.).

142 Vgl. BVerfGE 103, 89 (100).

143 Vgl. BVerfGE 89, 214 (232); 103, 89 (100 f.); 114, 1 (34); 73 (90); BVerfGK 8, 126 (131). 


\section{Das Verhältnis von Agenturen und Hochschulen}

Die Ausgestaltung des Verhältnisses von Agenturen zu den Hochschulen lässt sich nur anhand der gesamten Regelungsstruktur sachgerecht beurteilen. Überblickt man die bisherigen Aussagen dazu, wird man feststellen müssen, dass das Verhältnis von Agenturen zu Hochschulen nach wie vor höchst umstritten ist. Dies gilt vor allem für die Frage, ob die Agenturen privatrechtlich handeln und ob sie als Beliehene wirken und wenn ja, in welchen Formen. ${ }^{144}$ Dies hängt nicht zuletzt mit dem Verständnis der Akkreditierung zusammen.

\section{a. Akkreditierung als qualitative Bewertung}

Fast alle Hochschulgesetze enthalten heute ausführliche Regelungen zur Qualitätssicherung in Forschung, Lehre, Studium und Verwaltung, die regelmäßig mit interner wie externer Evaluation verbunden werden. Der gesetzliche Auftrag mündet vielfach in der Verpflichtung zum Aufbau eines internen Qualitätssicherungssystems, das in regelmäßigen Abständen extern evaluiert werden soll. ${ }^{145}$ Dies entspricht der oben dargestellten Verlagerung der Wahrnehmung der Gewährleistungsverantwortung für die Qualität von Studium und Lehre in die Hochschulen selbst. ${ }^{146}$ In diesem Kontext finden sich dann vielfach Regelungen über die Akkreditierung und Re-Akkreditierung von Studiengängen und ggf. ihrer Ersetzung durch akkreditierte Qualitätssicherungssysteme. ${ }^{147}$ Aus diesem Kontext heraus wird verständlich, dass eine erhebliche Zahl der Landesgesetze, selbst dann, wenn sie nicht explizit die Akkreditierung in diesem Kontext schon regeln, von der Akkreditierung als qualitative Bewertung sprechen, ${ }^{148}$ zum Teil werden anerkannte Qualitätsstandards ausdrücklich erwähnt. ${ }^{149}$ Auch wenn durchaus Elemente von Compliance darin enthalten sind, indem zudem auf die gesetzlichen Voraussetzungen oder die ländergemeinsamen Strukturvorgaben als Maßstab verwiesen wird, so geht es insoweit ersichtlich darum, eine qualitative Bewertung des jeweiligen Angebots (oder des Systems) vornehmen zu lassen. Insoweit ist dies, jedenfalls nach Auffassung der großen Mehrheit der Landesgesetze, nichts anderes als das, was auch schon im Bereich der Forschung an Bewertungsmechanismen etabliert ist: eine vom Land und den Hochschulen unabhängige Bewertung der Qualität, also im Grunde nichts anders als ein Unterfall der Evaluation.

144 Eine ausführliche Analyse findet sich bei D. Immer, Rechtsprobleme (Fn. 10), S. $167 \mathrm{ff}$.

145 Vgl. mit Nuancen im Einzelnen $\ 5$ LHG BW; $\int 8$ a BerlHG; $\$ 12$ HSchulG Hess; $\int 3$ Abs. 3 HmbHG;

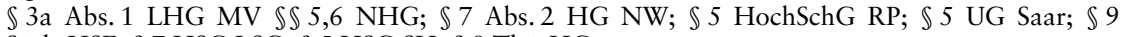
SächsHSF; $\mathbb{} 7$ HSG LSG; $\mathbb{} 5$ HSG SH; $\mathbb{} 8$ ThürHG.

146 Vgl. dazu oben C.I.1.

$147 \mathbb{\int} 8$ a Abs. 2 BerlHG; $\mathbb{S} 12$ Abs. 2 HSchulG Hess; $\mathbb{3}$ Abs. 4 LHG MV $\mathbb{} 6$ Abs. 2 NHG; $\mathbb{} 7$ Abs. 1 HG NN; $\mathbb{S} 5$ V HochSchG RP; $\mathbb{9}$ III SächsHSFG; $\mathbb{9}$ IV HSG LSA; $\mathbb{5}$ II HGS SH.

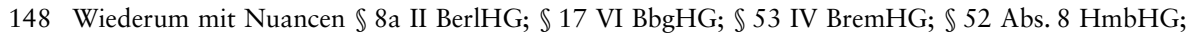

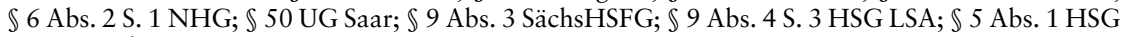
$\mathrm{SH} ; \mathbb{\int} 43$ ThürHG.

149 Vgl. etwa $\int 8$ a Abs. 2 S. 2 BerlHG. 
Von daher ist es auch gar nicht verwunderlich, dass in einer Reihe von Ländern die Akkreditierung nicht etwa die Genehmigung von Studiengängen oder deren Vereinbarung in Zielvereinbarungen ersetzt. Stattdessen steht sie neben diesen, ergänzt sie, wird als Voraussetzung einer solchen angesehen, oder sie wird mit einer Anzeigepflicht verbunden, wobei nach Zeitablauf ohne Widerspruch die Genehmigung fingiert wird. ${ }^{150}$ Aber auch in diesen Ländern ändert dies ersichtlich nichts an dem Charakter der Akkreditierung als qualitative Bewertung des Studienangebots. Der Nachweis der Qualität, der durch Akkreditierung erbracht wird, lässt sich in diesen Ländern als Verzicht des Staates auf eine Genehmigung verstehen. Dieser sieht seine Interessen, wie zum Beispiel die Ausfinanzierung von Studienangeboten, durch andere Instrumente, etwa über Zielvereinbarungen oder über Zustimmungsrechte des Hochschulrates, gesichert. Für die Systemakkreditierung gilt dies ohnehin. Der Aufbau von Qualitätssicherungssystemen war niemals Gegenstand von Genehmigungen und hier unterscheidet sich die Akkreditierung praktisch gar nicht von Zertifizierungen in anderen Feldern.

\section{b. Das Erfordernis einer gemeinwohlfähigen Verfassung der Kooperation}

Qualitative Bewertungen gibt es im Wissenschaftssystem vielfältig. Nicht selten werden an sie weitreichende Folgen, etwa hinsichtlich der Mittelvergabe, geknüpft. Man denke nur an Entscheidungen der DFG. Das Wissenschaftssystem ist insofern immer schon ein Feld der Kooperation und Bildung von hybriden institutionellen Arrangements gewesen, ${ }^{151}$ die stets die Schwierigkeiten der Zuordnung zum öffentlichen und privaten Recht aufgeworfen haben. Der Sache nach finden sich hier vielfältige Kooperationsformen, bei denen schon aus Gründen der Wissensverteilung die Aufgaben ohne Beteiligung privater Grundrechtsträger kaum sinnvoll erfüllt werden können. Hier hat sich früh gezeigt, dass die vorgeblich klaren Zäsuren nicht tragen, sondern jenseits dieser gemeinwohlfähige Voraussetzungen zu entwickeln sind, um die hybriden Arrangements verfassungs- und verwaltungsrechtlich angemessen zu verfassen. Die Einrichtung der Akkreditierung erfolgte um eines gewissen Maßes der Entstaatlichung von Qualitätssicherungsurteilen willen, um eine stärkere Differenzierung von Angeboten unter dem Stichwort der Profilbildung zu erreichen und eine Einbettung in einen europäischen Zusammenhang der Weiterentwicklung von Qualitätsstandards und Instrumenten zu befördern. Gewiss, auch diese hybriden Formen bedürfen einer angemessenen rechtlichen Durchformung. Aber diese muss, nach so vielen Jahren der Diskussion über den Formenwandel des Staatlichen, der Koopera-

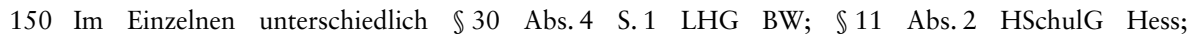

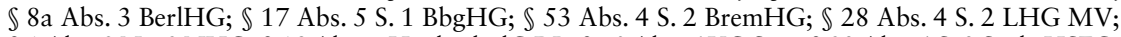

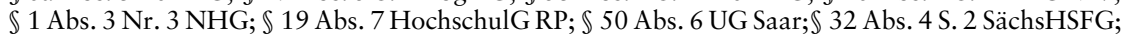
$\$ 49$ Abs. 6 HSG SH.

151 Vgl. dazu H.-H. Trute, Die Forschung zwischen grundrechtlicher Freiheit und staatlicher Institutionalisierung, 1994, S. 307 ff.; E. Schmidt-Aßmann, Ordnungsidee (Fn. 7), 3. Kap. Rn. 36 ff. 
tion von Staat und privaten Akteuren, keineswegs in der Etatisierung dieser institutionellen Arrangements liegen. ${ }^{152}$

So werden mit der Beleihung eines Rechtssubjekts zwar öffentlich-rechtliche Bindungen transportiert, gleichwohl sind damit die Probleme, die sich mit der Einbeziehung Privater in die staatliche Aufgabenerfüllung ergeben, noch nicht gelöst. Die dahin gehende Annahme verdeckt oft genug die Probleme, die sich aus der Einbeziehung Privater in die staatliche Aufgabenerfüllung ergeben. Diese können eben gerade darin liegen, dass eine juristische Person mit anderen kognitiven Orientierungen, Interessen, organisatorischen und personellen Ressourcen Aufgaben erledigen soll. ${ }^{153}$ Darin liegt ebenso sehr der Vorteil wie das Problem von Formen der Kooperation. ${ }^{154}$ Folgerichtig sind auch Anforderungen an die Ausgestaltung solcher institutioneller Arrangements zu formulieren, die dieser Situation Rechnung tragen. ${ }^{155}$ Denn ersichtlich wird der einbezogene Private durch den Staat in eine Entscheidungsposition gebracht und damit über die ansonsten vermutete Gleichordnung mit Privaten herausgehoben, also eine Form von private interest government konzipiert. ${ }^{156}$

\section{c. Die überwirkende Legitimationsverantwortung}

Die damit aufgeworfenen Probleme werden unter dem Stichwort der überwirkenden Legitimationsverantwortung diskutiert. Sie ist darauf gerichtet, die Gemeinwohlanforderungen auch dort sicherzustellen, wo eine Zusammenarbeit von staatlichen und privaten Akteuren stattfindet, ${ }^{157}$ soll also das hinreichende Maß an Legitimation in den Kooperationsstrukturen selbst garantieren. ${ }^{158}$ Der Schutzdimension der Grundrechte nicht unähnlich, ist insbesondere dem Gesetzgeber, aber auch der Verwaltung - etwa bei der vertraglichen Einbeziehung Privater in die Aufgabenerledigung - auf-

152 Vgl. dazu allgemein zur Figur der Beleihung E.Schmidt-Aßmann, Ordnungsidee (Fn. 7), 5. Kap. Rn. 57; M. Burgi, Der Beliehene - ein Klassiker im modernen Verwaltungsrecht, in: FS Hartmut Maurer, 2001, S. 581 (588 ff.); C. D. Classen, Demokratische Legitimation im offenen Rechtsstaat, Tübingen 2009, S. 71 ff.; H. Schulze-Fielitz, Aufgabenwahrnehmung (Fn. 1), Rn. 106; Th. Groß, Die Verwaltungsorganisation als Teil organisierter Staatlichkeit, in: W Hoffmann-Riem/E. Schmidt-Aßmann/A. Voßkuhle (Hrsg.), Grundlagen (Fn. 1), $\mathbb{1 3}$ Rn. 89 f.; H.-H. Trute, Legitimation (Fn. 7), Rn. 92.

153 H. Schulze-Fielitz, Aufgabenwahrnehmung (Fn. 1), Rn. 106.

154 Vgl. dazu H.-H. Trute, Legitimation (Fn. 7), \$6 Rn. 89 ff.

155 Deutlich BremStGH, NVwZ 2003, S. $81 \mathrm{ff}$.

156 Die Konstellationen sind freilich unterschiedlich, je nachdem, ob den Handlungen/Entscheidungen Privater noch eine staatliche Entscheidung nachgeordnet ist oder nicht. Zu dieser Unterscheidung und den unterschiedlichen Anforderungen vgl. H.-H. Trute, Legitimation (Fn. 7), \$ 6 Rn. 58.

157 H. Schulze-Fielitz, (Fn. 1), $\mathbb{S} 12$ Rn. $148 \mathrm{ff}$.

158 Mit Nuancen im Einzelnen H.-H. Trute, Wechselseitige Verzahnungen zwischen Privatrecht und öffentlichem Recht, in: W. Hoffmann-Riem/E. Schmidt-Aßmann (Hrsg.), Öffentliches Recht (Fn. 1), S. 167 (197 ff.); ders., Funktionen der Organisation (Fn. 68), S. 290; E.Schmidt-Aßmann, Ordnungsidee (Fn. 7), 2. Kap. Rn. 101 f., 5. Kap. Rn. 60; H. C. Röhl, Staatliche Verantwortung in Kooperationsstrukturen, DV 29 (1996), S. 487 ff.; Th. Groß, Kollegialprinzip (Fn. 94), S. 233 ff.; M. Burgi, Privatisierung (Fn. 1), S. 378 ff.; M. Fehling, Verwaltung zwischen Unparteilichkeit und Gestaltungsaufgabe, Tübingen 2001, S. 351 ff.; vgl. auch C.D. Classen, Legitimation (Fn. 159), S. 72 ff. 
gegeben, die institutionellen Rahmenbedingungen der Kooperation so zu gestalten, dass ein hinreichend gemeinwohlorientiertes Ergebnis erwartet werden kann. ${ }^{159}$ Ähnliches kann sich, ergänzend oder unterstützend, aus den objektiv-rechtlichen Dimensionen der Grundrechte ergeben. ${ }^{160}$ Die Anforderungen können die Ausgestaltung der Aufsicht und Kontrolle betreffen, die faktisch und normativ wirksam sein muss, um die sachgerechte, d. h. den rechtsstaatlichen und demokratischen Anforderungen entsprechende Aufgabenerledigung gemäß der für die übertragene Aufgabe geltenden Maßstäbe sicherzustellen. Über die klassischen Aufsichtsmittel hinaus können etwa aufgabenspezifische Kooperationsverträge oder die Schaffung von eigenständigen Kooperationsorganen in Betracht kommen. Darüber hinaus aber sind - je nach Aufgabenfeld unterschiedlich - Anforderungen zu stellen, die Interessenkonflikte ausschließen, also hinreichende Neutralitätssicherungen abgeben. Hierzu können Transparenzgebote und institutionelle Unabhängigkeitssicherungen gegenüber anderen Aufgabenfeldern der Organisation treten.

\section{Anforderungen an das Kooperationsspektrum im Akkreditierungsbereich}

Man kann die Anforderungen typisierend zusammenfassen als Gebote sachgerechter Aufgabenwahrnehmung, gleichmäßiger Interessenberücksichtigung und hinreichender institutioneller Neutralitätssicherung. ${ }^{161}$

\section{a. Sachgerechte Aufgabenwahrnehmung}

Die Anforderung der sachgerechten Aufgabenwahrnehmung findet ihren Grund in der fortbestehenden Strukturverantwortung des Staates bei gleichzeitiger Einbeziehung privater Akteure. Dies wird man dergestalt beschreiben können, dass die staatlichen Qualitätsziele und die Verfahren zu ihrer Erreichung, die in den Maßstäben der Akkreditierung von Studiengängen, Qualitätssicherungssystemen und Einrichtungen niedergelegt werden, ${ }^{162}$ auch durch die privaten Akteure beachtet werden. Dabei hat die obige Analyse gezeigt, dass insoweit nicht nur eine detaillierte materielle und prozedurale Konkretisierung der Anforderungen besteht, sondern ebenso eine intensive und wiederholte Prüfung der Agenturen daraufhin, ob diese von ihren eigenen Strukturen und Ressourcen her geeignet sind, die Aufgaben zu erfüllen. Ergänzt wird dies durch die auch während der Akkreditierung möglichen Nachsteuerungen, wie auch durch die Berichtspflichten hinsichtlich erfolgter Akkreditierungen und deren Versagungen unter Angabe von Gründen, die zudem mit Publizitätspflichten verbunden sind. Dies wiederum wird durch die Aufsicht des Akkreditie-

159 H.-H. Trute, Legitimation (Fn. 7), Rn. 89 ff.

160 Vgl. bereits H.-H. Trute, Die Forschung (Fn. 158), S. 315 ff., sowie oben C.)I.)1.).

161 Vgl. dazu ausführlich bereits H.-H. Trute, Verzahnungen (Fn. 167), S. 167 (208 ff.).

162 Dabei handelt es sich sowohl um staatliche erlassene Rechtsnormen wie auch durch den Akkreditierungsrat weiter konkretisierte materielle und prozedurale Vorgaben; zu den insoweit gleichwohl bestehenden Strukturierungsdefiziten vgl. oben C.) III.) 1.) c.). 
rungsrates über die Agenturen komplementiert. Angesichts der Tatsache, dass damit vielfältige Mittel bestehen, um nicht nur punktuell und nicht nur im Versagensfalle auf die Agenturen zu einer sachgerechten Zielerreichung einzuwirken, wird auch hinsichtlich der Agenturen ein Qualitätssicherungsverfahren begründet, das zum einen als ein auch internationale Erfahrungen fortlaufend berücksichtigendes Kooperationsverhältnis konzipiert ist und zum anderen die Erfahrungen im Umgang mit den Aufgaben bei den nach Ablauf der Zeit erforderlichen Re-Akkreditierungen zu berücksichtigen erlaubt. So lassen sich etwa im Verfahren der Systemakkreditierung verschärfte Vorschriften zur sachgerechten Besetzung der Gutachtergruppen als Reaktion auf Erfahrungen deuten, die von großen Universitäten im Hinblick auf eine sachgerechte Einschätzung von Managementanforderungen dieser Einrichtungen erkannt wurden. ${ }^{163}$ Man kann nicht recht sehen, dass diese Qualitätssicherungsvorkehrungen in gleicher Weise etwa in klassischen Fällen der Beleihung vorgehalten werden, noch kann man - weitergehend - einfach unterstellen, dass der Staat in vergleichbaren Fällen auch vergleichbare Standards gegen sich selbst garantiert.

\section{b. Hinreichende Neutralitätssicherungen}

Im Ergebnis gilt nichts anderes hinsichtlich der Neutralitätssicherungen. Durch die Konstruktion des Akkreditierungsrates und dessen Zusammensetzung ist zunächst einmal gesichert, dass eine direkte Abhängigkeit von staatlichen Hochschulverwaltungen nicht besteht. ${ }^{164}$ Dies erlaubt eine von den direkten und nicht unbedingt transparenten Zugriffen freie und insofern durchaus einheitliche Handhabung des Akkreditierungsgeschehens.

Darüber hinaus sind die Agenturen von den Hochschulen als Auftraggebern der Akkreditierung unabhängig zu halten. Insoweit ist jedenfalls das Verfahren so ausgestaltet, dass die Hochschulen gegen bestellte Gutachter Einspruchsrechte haben wenn auch sinnvoller Weise keine Vorschlags- oder Vetorechte. ${ }^{165}$ Dies entspricht dem allgemeinen Stand von peer-review-Verfahren in der Wissenschaft.

Eine weitere Schicht der Unabhängigkeitsgarantien besteht in der Verpflichtung auf die Lauterbarkeit der Siegelvergabe. Sie soll das durch den Akkreditierungsrat gesteuerte Akkreditierungsgeschehen gegenüber institutionellen Eigeninteressen der Agenturen an der Vergabe anderer Siegel frei halten.

163 Vgl. dazu Wissenschaftsrat, Empfehlungen (Fn. 12), S. 58, 80 f.

164 Dies wird anders zu beurteilen sein, wenn - wie etwa in dem Entwurf des Hochschulzukunftsgesetzes NRW - die staatlichen Hochschulverwaltungen sich auf der Grundlage von Beleihungstatbeständen direkte Zugriffsrechte auf die Agenturen sichern. Damit ist jedenfalls eine operative Unabhängigkeit der Agenturen nicht mehr gegeben.

165 Regeln für die Akkreditierung von Studiengängen und für die Systemakkreditierung v. 8.12.2009, zuletzt geändert am 20.3.2013, Drs. AR 20/2013 Ziff. 1.1.3. 


\section{c. Hinreichende Interessenberücksichtigung}

Darüber hinaus bedarf es sicherlich eines Verfahrens, das die Interessen der Universität in Bezug auf den zu akkreditierenden Studiengang oder das Qualitätsmanagementsystem gewährleistet. Insoweit weisen die Regelungen darauf hin, dass die Hauptverantwortung für die Konzeption und Qualität zunächst einmal bei den Hochschulen liegt. Kritik an der Usurpation von Entscheidungsspielräumen ist durch die Nachsteuerung der Anforderungen Rechnung getragen worden. ${ }^{166}$ Darüber hinaus ist von erheblicher Bedeutung, dass das Verfahren auf einer Selbstbeschreibung des Studiengangs oder Qualitätsmanagementsystems durch die Hochschulen anhand der genannten Maßstäbe aufbaut. ${ }^{167}$ Dies sichert die Herrschaft der Hochschulen über den Gegenstand und gewährleistet damit im Rahmen der Maßstäbe die autonome Gestaltung des Studiengangs oder Managementsystems, die von einer umfassenden Beratung im Vorfeld der Erstellung der Selbstbeschreibung begleitet wird. Gleichwohl wird die Hochschule einem externen Qualitätsurteil ausgesetzt, was der Sache nach weder zu beanstanden noch für den Wissenschaftsbereich ungewöhnlich ist. Der peer review setzt allerdings eine hinreichende Kompetenz der peers voraus. Diese soll zum einen durch eine den betroffenen Interessen (Wissenschaft, Studierende, Berufspraxis) Rechnung tragende Besetzung gesichert werden. ${ }^{168}$ Darüber hinaus sehen die Regeln eine angemessene Vorbereitung der peers auf das Begehungsverfahren vor. ${ }^{169}$ Dazu gehört nicht nur eine Vertrautheit mit dem Gegenstand und eine entsprechende Kompetenz bezüglich desselben, sondern auch und vor allem eine gewisse Rollendistanz, die die Autonomie der Hochschulen achtet und nicht deren Gestaltungspielräume usurpiert - nach allen Erfahrungen eine jedenfalls nicht fernliegende Möglichkeit. Die Anforderungen werden zum Teil für die Verfahren noch spezifiziert, wie etwa im Kontext der Systemakkreditierung, in der etwa ein Mitglied über umfassende Erfahrung in der Hochschulleitung, Studiengangsgestaltung und in der Qualitätssicherung von Studium und Lehre verfügen soll. ${ }^{170}$ Insoweit wird den Anforderungen an die Kompetenz von peers in diesem Verfahren durchaus umfassend Rechnung getragen. Die Transparenz wird durch Verfahrensgestaltung zusätz-

166 Dazu Wissenschaftsrat, Empfehlungen (Fn. 12), S. 52 f.

167 Die zu beachtenden Maßstäbe sind noch einmal in den Regeln für die Akkreditierung von Studiengängen und für die Systemakkreditierung v. 8.12.2009, zuletzt geändert am 20.3.2013, Drs. AR 20/2013 Ziff 2.2 unter dem Stichwort der Einordnung des Studiengangs in das Studiensystem zusammengefasst. Darüber hinaus werden die Kriterien insgesamt zusammengefasst unter 2. der Regeln bezogen auf die Studiengangsziele, konzeptionelle Einordnung, das Studiengangskonzept, Studierbarkeit, Prüfungssystem, Kooperationen, Transparenz, Qualitätssicherung und Weiterentwicklung, besonderen Profilanspruch und Genderaspekte einschließlich der Chancengleichheit.

168 Regeln für die Akkreditierung von Studiengängen und für die Systemakkreditierung v. 8.12.2009, zuletzt geändert am 20.3.2013, Drs. AR 20/2013 1.1.3.

169 Vorbereitung von Gutachterinnen und Gutachtern in Akkreditierungsverfahren, Beschluss des AR v. 31.10.2008, Drs. AR 88/2008.

170 Regeln für die Akkreditierung von Studiengängen und für die Systemakkreditierung v. 8.12.2009, zuletzt geändert am 20.3.2013, Drs. AR 20/2013 5.5. 
lich gesichert, indem Gespräche mit allen relevanten Interessen im Rahmen des peer reviews stattfinden und zudem die Eindrücke eigenständig formuliert und kommentiert werden können. Darüber hinaus kann die Hochschule zu dem Gutachten der peers Stellung nehmen, bevor die Entscheidung der Agentur getroffen wird. Einspruchsrechte sind ebenfalls gesichert. Insoweit wird man das hier nur grob dargestellte Verfahren jedenfalls nicht für ungeeignet halten dürfen, die Interessen der Einrichtungen hinreichend zu berücksichtigen und - sachgerecht durchgeführt - auch die Autonomieansprüche der Einrichtungen zu wahren. ${ }^{171}$ Strukturell dürfte es jedenfalls in Sachen Kompetenz und Transparenz schwerlich hinter rein staatlichen Verfahren der Genehmigung zurückbleiben. Allerdings käme bei dem derzeitigen Stand der Qualitätsdiskussion keine Wissenschaftsadministration ohne diese Formen von peer review aus - schon mangels hinreichender kognitiver Ressourcen und schon gar nicht könnte sie die Dynamik der Qualitätsentwicklung anders als durch eine vergleichbare Struktur sicherstellen.

Vor diesem Hintergrund verliert der Streit um die Frage der Beleihung viel von seiner Bedeutung. Denn - richtig verstanden - unterscheiden sich die Anforderungen in der Sache letztlich wenig. Insoweit ginge es freilich - unabhängig davon welcher Konzeption man folgt - um eine entsprechende Ausgestaltung der Kooperationsstruktur. Wohl aber macht es Unterschiede im Hinblick auf die angestrebte Internationalisierung und für die einbezogenen Privaten. Denn deren Einbeziehung in eine öffentlichrechtliche Struktur erweist sich als eine Form der Etatisierung, die schwerlich mit ihren Gründungsintentionen übereinstimmt und die die Entwicklung der Qualitätsdiskussion eher behindern als befördern würde.

\section{Schluss}

Es gibt vielfältige Formen der Einbeziehung Privater in den Vollzug öffentlich-rechtlicher Normen, Standards und Entscheidungen. Die Etablierung einer Akkreditierungs- und Zertifizierungsinfrastruktur, die sich als ein hybrides Arrangement von staatlichen, intermediären und privaten Akteuren darstellt, ist - wie letztlich schon ein Vergleich mit anderen Wissenschaftssystemen zeigt - weder eine überflüssige Parallelstruktur noch könnte sie sinnvoll durch eine staatliche Administration ersetzt werden. Über ihre Qualitätssicherungsfunktion hinaus hat sie eine wichtige Funktion darin, Wissen über Qualitätssicherung im Wissenschaftsbereich zu generieren und die Standards möglichst mit internationalen Entwicklungen in diesem Bereich kompatibel zu halten. Sie folgt damit anderen Bereichen transnationaler Vernetzung,

171 Die empirischen Untersuchungen sind insoweit durchaus instruktiv als aus der Perspektive vieler Hochschulleitungen, Dekanate und Studierenden die Akkreditierung eine Anreizstruktur darstellt, sich mit Fragen der Konzeption von Studiengängen und ihrer Qualität zu befassen und insofern als hilfreich empfunden wird. Diese Sicht wird naturgemäß auf der Ebene der Lehrenden zumindest nicht durchgängig geteilt; vgl. dazu B. Kaufmann, Akkreditierung als Mikropolitik. Zur Wirkung neuer Steuerungsinstrumente an deutschen Hochschulen, Wiesbaden 2012, S. 105 ff. 
auch wenn diese deutlich geringer ausgeprägt sein mag, als in anderen Bereichen. Diese Funktionen können schwerlich von einer Länderadministration sinnvoll wahrgenommen werden. Vielmehr wird in gewisser Weise diese Struktur zu einem wichtigen Teil der Qualitätsverantwortung des Staates. Von daher ist die Frage der angemessenen Ausgestaltung alles andere als überflüssig. Sie erschöpft sich freilich nicht in einfachen Formeln und medial befeuerten Träumen von der Rückkehr zu Zuständen, die als gut nur derjenige beurteilen kann, der nie mit staatlichen Hochschuladministrationen kleinteilige Bedenken über die Genehmigung von Studiengängen verhandelt hat. Der Beweis dafür, dass das ein transparenteres und wissenschaftsadäquateres System gewesen sei, der steht noch aus. Dies muss die Weiterentwicklung des Systems der Akkreditierung ebenso wenig behindern, wie es ein Mandat ist, rechtsstaatliche und demokratische Defizite der Struktur nicht zu beseitigen. 